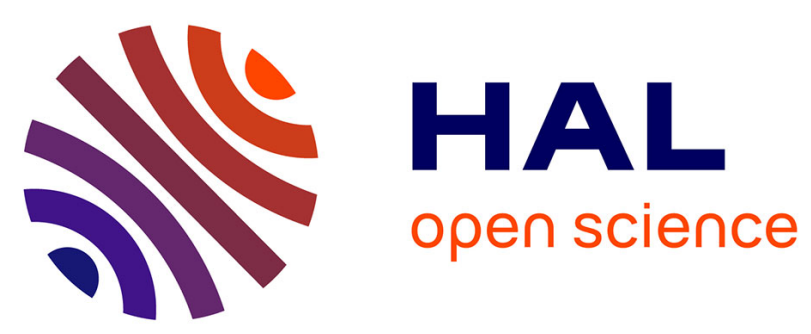

\title{
Theoretical Performance of Low Rank Adaptive Filters in the Large Dimensional Regime
}

Alice Combernoux, Frédéric Pascal, Guillaume Ginolhac, Marc Lesturgie

\section{To cite this version:}

Alice Combernoux, Frédéric Pascal, Guillaume Ginolhac, Marc Lesturgie. Theoretical Performance of Low Rank Adaptive Filters in the Large Dimensional Regime. IEEE Transactions on Aerospace and Electronic Systems, 2019, 27 (6), pp.3347 - 3364. 10.1109/TAES.2019.2906418 . hal-02083430

\section{HAL Id: hal-02083430 https://hal.science/hal-02083430}

Submitted on 26 Feb 2020

HAL is a multi-disciplinary open access archive for the deposit and dissemination of scientific research documents, whether they are published or not. The documents may come from teaching and research institutions in France or abroad, or from public or private research centers.
L'archive ouverte pluridisciplinaire HAL, est destinée au dépôt et à la diffusion de documents scientifiques de niveau recherche, publiés ou non, émanant des établissements d'enseignement et de recherche français ou étrangers, des laboratoires publics ou privés. 


\title{
Theoretical Performance of Low Rank Adaptive Filters in Gaussian Context in the Large Dimensional Regime
}

\author{
Alice Combernoux, Student Member, IEEE, Frédéric Pascal, Senior Member, IEEE, \\ Guillaume Ginolhac, Member, IEEE and Marc Lesturgie Senior Member, IEEE
}

\begin{abstract}
This paper addresses the problem of deriving the asymptotic performance of adaptive Low Rank (LR) filters used in target detection embedded in a disturbance composed of a LR Gaussian noise plus a white Gaussian noise. In this context, we use the Signal to Interference to Noise Ratio (SINR) loss as performance measure which is a function of the estimated projector onto the LR noise subspace. However, although the SINR loss can be determined through Monte-Carlo simulations or real data, this process remains quite time consuming. Thus, this paper proposes to predict the SINR loss behavior in order to not depend on the data anymore and be quicker. To derive this theoretical result, previous works used a restrictive hypothesis assuming that the target is orthogonal to the LR noise. In this paper, we propose to derive this theoretical performance by relaxing this hypothesis and using Random Matrix Theory (RMT) tools. These tools will be used to present the convergences of simple quadratic forms and perform new RMT convergences of structured quadratic forms and SINR loss in the large dimensional regime, i.e. the size and the number of the data tend to infinity at the same rate. We show through simulations the interest of our approach compared to the previous works when the restrictive hypothesis is no longer verified.
\end{abstract}

Index Terms-Low Rank SINR loss, Random Matrix Theory, Adaptive Filtering, Quadratic Forms convergence, Spiked model

\section{INTRODUCTION}

In array processing, the covariance matrix $\mathbf{R}$ of the data is widely involved for main applications as filtering [1], [2], radar/sonar detection [3] or localization [4], [5]. However, when the disturbance in the data is composed of the sum of a Low Rank (LR) correlated noise and a White Gaussian Noise (WGN), the covariance matrix is often replaced by the projector onto the LR noise subspace $\boldsymbol{\Pi}_{\mathrm{c}}$ [6]-[9]. In practice, the projector onto the LR noise subspace (and the covariance matrix) is generally unknown and an estimate is consequently required to perform the different processing. This estimation procedure is based on the so-called secondary data assumed to be independent and to share the same distribution. Then, the true projector is replaced by the estimated one in order to obtain an adaptive processing. An important issue is then to derive the theoretical performance of the adaptive processing as a function of the number of secondary data $K$. The processing based on the covariance matrix has been widely

Alice Combernoux and Marc Lesturgie are with SONDRA - ONERA, CentraleSupelec, Frédéric Pascal is with L2S - CentraleSupelec - CNRS Université Paris-Sud 11, Guillaume Ginolhac is with LISTIC - Université Savoie Mont Blanc studied and led to many theoretical results in filtering [1] and detection [10]-[13]. For example, for classical adaptive processing, $K=2 m$ secondary data (where $m$ is the data size) are required to ensure good performance of the adaptive filtering, i.e. a $3 \mathrm{~dB}$ loss of the output Signal to Interference plus Noise Ratio (SINR) compared to optimal filtering [1]. For LR processing, some results has been obtained especially in filtering [6], [14]-[16] and localization [17]. Similarly, in LR filtering, the number $K$ of secondary data required to ensure good performance of the adaptive filtering is equal to $2 r$ (where $r$ is the rank of the LR noise subspace) [6], [14].

These last results are obtained from the theoretical study of the SINR loss. More precisely, in [14], [16], the derivation of the theoretical results is based on the hypothesis that the steering vector is orthogonal to the LR noise subspace. Nevertheless, even if the result seems to be close to the simulated one, when the hypothesis is no longer valid anymore [18], it is impossible with traditional techniques of [14], [16] to obtain a theoretical performance as a function of the distance between the steering vector and the LR noise subspace. Since, in practice, this dependence is essential to predict the performance of the adaptive filtering, we propose in this paper to derive the theoretical SINR loss, for a disturbance composed of a LR Gaussian noise and a WGN, as a function of $K$ and the distance between the steering vector and the LR noise subspace. The proposed approach is based on the study of the SINR loss structure.

The SINR loss (resp. LR SINR loss) is composed of a simple Quadratic Form (QF) in the numerator, $\boldsymbol{s}_{1}^{H} \hat{\mathbf{R}}^{-1} \boldsymbol{s}_{2}$ (resp. $\boldsymbol{s}_{1}^{H} \hat{\boldsymbol{\Pi}}_{\mathrm{c}}^{\perp} \boldsymbol{s}_{2}$ ) and a structured $\mathrm{QF}$ in the denominator $\boldsymbol{s}_{1}^{H} \hat{\mathbf{R}}^{-1} \mathbf{R}^{-1} \boldsymbol{s}_{2}$ (resp. $\boldsymbol{s}_{1}^{H} \hat{\boldsymbol{\Pi}}_{\mathrm{c}}^{\perp} \mathbf{R} \hat{\boldsymbol{\Pi}}_{\mathrm{c}}^{\perp} \boldsymbol{s}_{2}$ ). These recent years, the simple QFs (numerator) have been broadly studied [19][22] using Random Matrix Theory (RMT) tools contrary to structured QFs (denominator). RMT tools have also been used in array processing to improve the MUSIC algorithm [23], [24] and in matched subspace detection [25], [26] where the rank $r$ is unknown. The principle is to examine the spectral behavior of $\hat{\mathbf{R}}$ by RMT to obtain their convergences, performance and asymptotic distribution when $K$ tends to infinity and when both the data size $m$ and $K$ tend to infinity at the same ratio, i.e. $m / K \rightarrow c \in] 0,+\infty)$, for different models of $\hat{\mathbf{R}}$ of the observed data as in [19], [20], [23], [22] and [21]. Therefore, inspired by these works, we propose in this paper to summarize the convergences of the structured QFs $\boldsymbol{s}_{1}^{H} \hat{\mathbf{R}}^{-1} \mathbf{R} \hat{\mathbf{R}}^{-1} \boldsymbol{s}_{2}$ and study those of $\boldsymbol{s}_{1}^{H} \hat{\boldsymbol{\Pi}}_{\mathrm{c}}^{\perp} \mathbf{R} \hat{\boldsymbol{\Pi}}_{\mathrm{c}}^{\perp} \boldsymbol{s}_{2}$ : 
when 1) $K \rightarrow \infty$ with a fixed $m$ and when 2) $m, K \rightarrow \infty$ at the same ratio under the most appropriated model for our data and with the rank assumed to be known. From [27], [28], the spiked model has proved to be the more appropriated one to our knowledge. This model, introduced by [29] (also studied in [30], [31] from an eigenvector point of view) considers that the multiplicity of the eigenvalues corresponding to the signal (the LR noise in this article) is fixed for all $m$ and leads to the SPIKE-MUSIC estimator [32] of $\boldsymbol{s}_{1}^{H} \hat{\boldsymbol{\Pi}} \boldsymbol{s}_{2}$. Then, the new results are validated through numerical simulations. From these new theoretical convergences, the paper derives the convergence of the LR SINR loss for LR filters. The new theoretical SINR losses depend on the number of secondary data $K$ but also on the distance between the steering vector and the LR noise subspace. This work is partially related to those of [33]-[36] and [37] which uses the RMT tools to derive the theoretical SINR loss or SNR performance in a full rank context (previously defined as classical).

Finally, this theoretical LR SINR loss is validated in a jamming application context where the purpose is to detect a target thanks to a Uniform Linear Antenna (ULA) composed of $m$ sensors despite the presence of jamming. The response of the jamming is composed of signals similar to the target response. This problem is very similar to the well-known Space Time Adaptive Processing (STAP) introduced in [2]. The results show the interest of our approach with respect to other theoretical results [6], [14]-[16] in particular when the target is close to the jammer.

The paper is organized as follows. Section II presents the received data model, the adaptive filters and the corresponding SINR losses. Section III summarizes the existing studies on the simple QFs $\boldsymbol{s}_{1}^{H} \hat{\mathbf{R}} \boldsymbol{s}_{2}$ and $\boldsymbol{s}_{1}^{H} \hat{\boldsymbol{\Pi}} \boldsymbol{s}_{2}$, and exposes the covariance matrix model, the spiked model. Section IV gives the theoretical contribution the paper with the convergences of the structured QFs $\boldsymbol{s}_{1}^{H} \hat{\boldsymbol{\Pi}}_{\mathrm{c}}^{\perp} \mathbf{B} \hat{\boldsymbol{\Pi}}_{\mathrm{c}}^{\perp} \boldsymbol{s}_{2}$ and $\boldsymbol{s}_{1}^{H} \hat{\boldsymbol{\Pi}}_{\mathrm{c}}^{\perp} \mathbf{R} \hat{\boldsymbol{\Pi}}_{\mathrm{c}}^{\perp} \boldsymbol{s}_{2}$ and the convergences of the LR SINR loss. The results are finally applied on a jamming application in Section V.

Notations: The following conventions are adopted. An italic letter stands for a scalar quantity, boldface lowercase (uppercase) characters stand for vectors (matrices) and $(.)^{H}$ stands for the conjugate transpose. $\mathbf{I}_{N}$ is the $N \times$ $N$ identity matrix, $\operatorname{tr}($.$) denotes the trace operator and$ $\operatorname{diag}($.$) denotes the diagonalization operator such as (\mathbf{A})_{i, i}=$ $(\operatorname{diag}(\mathbf{a}))_{i, i}=(\mathbf{a})_{i}$ and $(\mathbf{A})_{i, j}=0$ if $i \neq j . \#\{\mathcal{A}\}$ denotes the cardinality of the set $\mathcal{A}$. $\llbracket a, b \rrbracket$ is the set defined by $\left\{x \in \mathbb{Z}: a \leqslant x \leqslant b, \forall(a, b) \in \mathbb{Z}^{2}\right\} . \mathbb{D}_{n \times N}$ is a $n \times N$ matrix full of 0 . The abbreviations iid and a.s. stem for independent and identically distributed and almost surely respectively.

\section{Problem Statement}

The aim of the problem is to filter the received observation vector $\boldsymbol{x} \in \mathbb{C}^{m \times 1}$ in order to whiten the noise without mitigating an eventual complex signal of interest $\boldsymbol{d}$ (typically a target in radar processing). In this paper, $\boldsymbol{d}$ will be a target response and is equal to $\alpha \boldsymbol{a}(\Theta)$ where $\alpha$ is an unknown complex deterministic parameter (generally corresponding to the target amplitude), $\boldsymbol{a}(\boldsymbol{\Theta})$ is the steering vector and $\boldsymbol{\Theta}$ is an unknown deterministic vector containing the different parameters of the target (e.g. the localization, the velocity, the Angle of Arrival (AoA), etc.). In the remainder of the article, in order to simplify the notations, $\Theta$ will be omitted of the steering vector which will simply be denoted as $\boldsymbol{a}$. If necessary, the original notation will be taken.

This section will first introduce the data model. Then, the filters, adaptive filters and the quantity characterizing their performance, the SINR loss, will be defined.

\section{A. Data model}

The observation vector can be written as:

$$
\boldsymbol{x}=\boldsymbol{d}+\boldsymbol{c}+\boldsymbol{b}
$$

where $\boldsymbol{c}+\boldsymbol{b}$ is the noise that has to be whitened. $\boldsymbol{b} \in$ $\mathbb{C}^{m \times 1} \sim \mathcal{C N}\left(\mathbf{0}, \sigma^{2} \mathbf{I}_{m}\right)$ is an Additive WGN (AWGN) and $c \in \mathbb{C}^{m \times 1}$ is a LR Gaussian noise modeled by a zeromean complex Gaussian vector with a normalized covariance matrix $\mathbf{C}(\operatorname{tr}(\mathbf{C})=m)$, i.e. $\boldsymbol{c} \sim \mathcal{C N}(\mathbf{0}, \mathbf{C})$. Consequently, the covariance matrix of the noise $\boldsymbol{c}+\boldsymbol{b}$ can be written as $\mathbf{R}=\mathbf{C}+\sigma^{2} \mathbf{I}_{m} \in \mathbb{C}^{m \times m}$. Moreover, considering a LR Gaussian noise, one has $\operatorname{rank}(\mathbf{C})=r \ll m$ and hence, the eigendecomposition of $\mathbf{C}$ is:

$$
\mathbf{C}=\sum_{i=1}^{r} \omega_{i} \boldsymbol{u}_{i} \boldsymbol{u}_{i}^{H}
$$

where $\omega_{i}$ and $\boldsymbol{u}_{i}, i \in \llbracket 1 ; r \rrbracket$ are the non-zero eigenvalues and the associated eigenvectors of $\mathbf{C}$ respectively, unknown in practice. This leads to:

$$
\mathbf{R}=\sum_{i=1}^{m} \lambda_{i} \boldsymbol{u}_{i} \boldsymbol{u}_{i}^{H}
$$

where $\lambda_{i}$ and $\boldsymbol{u}_{i}, i \in \llbracket 1, m \rrbracket$ are the eigenvalues and the associated eigenvectors of $\mathbf{R}$ respectively with $\lambda_{1}=\omega_{1}+\sigma^{2}>$ $\cdots>\lambda_{r}=\omega_{r}+\sigma^{2}>\lambda_{r+1}=\cdots=\lambda_{m}=\sigma^{2}$. Then, the projector onto the LR Gaussian noise subspace $\boldsymbol{\Pi}_{\mathrm{c}}$ and the projector onto the orthogonal subspace to the LR Gaussian noise subspace $\Pi_{\mathrm{c}}^{\perp}$ are defined as follows:

$$
\left\{\begin{array}{l}
\boldsymbol{\Pi}_{\mathrm{c}}=\sum_{i=1}^{r} \boldsymbol{u}_{i} \boldsymbol{u}_{i}^{H} \\
\boldsymbol{\Pi}_{\mathrm{c}}^{\perp}=\mathbf{I}_{m}-\boldsymbol{\Pi}_{\mathrm{c}}=\sum_{i=r+1}^{m} \boldsymbol{u}_{i} \boldsymbol{u}_{i}^{H}
\end{array}\right.
$$

However, in practice, the covariance matrix $\mathbf{R}$ of the noise is unknown. Consequently, it is traditionally estimated with the Sample Covariance Matrix (SCM) which is computed from $K$ iid secondary data $\boldsymbol{x}_{k}=\boldsymbol{c}_{k}+\boldsymbol{b}_{k}, k \in \llbracket 1, K \rrbracket$ with $\boldsymbol{c}_{k} \sim$ $\mathcal{C N}(\mathbf{0}, \mathbf{C})$ and $\boldsymbol{b}_{k} \sim \mathcal{C N}\left(\mathbf{0}, \sigma^{2} \mathbf{I}_{m}\right)$, and can be written as:

$$
\hat{\mathbf{R}}=\frac{1}{K} \sum_{k=1}^{K} \boldsymbol{x}_{k} \boldsymbol{x}_{k}^{H}=\sum_{i=1}^{m} \hat{\lambda}_{i} \hat{\boldsymbol{u}}_{i} \hat{\boldsymbol{u}}_{i}^{H}
$$

where $\hat{\lambda}_{i}$ and $\hat{\boldsymbol{u}}_{i}, i \in \llbracket 1, m \rrbracket$ are the eigenvalues and the eigenvectors of $\hat{\mathbf{R}}$ respectively with $\hat{\lambda}_{1} \geqslant \hat{\lambda}_{2} \geqslant \cdots \geqslant \hat{\lambda}_{m}$. For simplicity purposes, we set $\sigma^{2}=1$. Finally, the traditional projectors estimators based on the SCM are:

$$
\left\{\begin{array}{l}
\hat{\boldsymbol{\Pi}}_{\mathrm{c}}=\sum_{i=1}^{r} \hat{\boldsymbol{u}}_{i} \hat{\boldsymbol{u}}_{i}^{H} \\
\hat{\boldsymbol{\Pi}}_{\mathrm{c}}^{\perp}=\mathbf{I}_{m}-\hat{\boldsymbol{\Pi}}_{\mathrm{c}}=\sum_{i=r+1}^{m} \hat{\boldsymbol{u}}_{i} \hat{\boldsymbol{u}}_{i}^{H},
\end{array}\right.
$$




\section{B. Adaptive filters}

A filtering preprocessing on the observation vector $\boldsymbol{x}$ (Eq.(1)) is first done with the filter $\boldsymbol{w}$ in order to whiten the received signal: $p=\boldsymbol{w}^{H} \boldsymbol{x}$. The filter maximizing the SINR is given by:

$$
\boldsymbol{w}_{\mathrm{opt}}=\mathbf{R}^{-1} \boldsymbol{a}
$$

Since, in practice, the covariance matrix $\mathbf{R}$ of the noise is unknown, the estimated optimal filter or adaptive filter (suboptimal) is:

$$
\hat{\boldsymbol{w}}=\hat{\mathbf{R}}^{-1} \boldsymbol{a}
$$

In the case where one would benefit of the LR structure of the noise, one should use the optimal LR filter, based on the fact that $\boldsymbol{\Pi}_{\mathrm{c}}^{\perp}$ is the best rank $r$ approximation of $\mathbf{R}^{-1}$, which is defined by [6]:

$$
\boldsymbol{w}_{\mathrm{LR}}=\boldsymbol{\Pi}_{\mathrm{c}}^{\perp} \boldsymbol{a}
$$

Since, in practice, the projector is not known and is estimated from the SCM, the estimated optimal filter or adaptive filter (sub-optimal) is:

$$
\hat{\boldsymbol{w}}_{\mathrm{LR}}=\hat{\boldsymbol{\Pi}}_{\mathrm{c}}^{\perp} \boldsymbol{a}
$$

\section{SINR Loss}

Then, we define the SINR Loss. In order to characterize the performance of the estimated filters, the SINR loss compares the SINR at the output of the filter to the maximum SINR:

$$
\begin{aligned}
\hat{\rho} & =\frac{S I N R_{\text {out }}}{S I N R_{\max }}=\frac{\left|\hat{\boldsymbol{w}}^{H} \boldsymbol{d}\right|^{2}}{\left(\hat{\boldsymbol{w}}^{H} \mathbf{R} \hat{\boldsymbol{w}}\right)\left(\boldsymbol{d}^{H} \mathbf{R}^{-1} \boldsymbol{d}\right)} \\
& =\frac{\left|\boldsymbol{a}^{H} \hat{\mathbf{R}}^{-1} \boldsymbol{a}\right|^{2}}{\left(\boldsymbol{a}^{H} \hat{\mathbf{R}}^{-1} \mathbf{R} \hat{\mathbf{R}}^{-1} \boldsymbol{a}\right)\left(\boldsymbol{a}^{H} \mathbf{R}^{-1} \boldsymbol{a}\right)}
\end{aligned}
$$

If $\hat{\boldsymbol{w}}=\boldsymbol{w}_{\mathrm{opt}}$, the SINR loss is maximum and is equal to 1 . When we consider the LR structure of the noise, the theoretical SINR loss can be written as:

$$
\begin{aligned}
\rho_{\mathrm{LR}} & =\frac{\left|\boldsymbol{w}_{\mathrm{LR}}^{H} \boldsymbol{d}\right|^{2}}{\left(\boldsymbol{w}_{\mathrm{LR}}^{H} \mathbf{R} \boldsymbol{w}_{\mathrm{LR}}\right)\left(\boldsymbol{d}^{H} \mathbf{R}^{-1} \boldsymbol{d}\right)} \\
& =\frac{\left|\boldsymbol{a}^{H} \boldsymbol{\Pi}_{\mathrm{c}}^{\perp} \boldsymbol{a}\right|^{2}}{\left(\boldsymbol{a}^{H} \boldsymbol{\Pi}_{\mathrm{c}}^{\perp} \mathbf{R} \boldsymbol{\Pi}_{\mathrm{c}}^{\perp} \boldsymbol{a}\right)\left(\boldsymbol{a}^{H} \mathbf{R}^{-1} \boldsymbol{a}\right)}
\end{aligned}
$$

Finally, the SINR loss corresponding to the adaptive filter in Eq.(10) is defined from Eq.(14) as:

$$
\hat{\rho}_{\mathrm{LR}}=\left.\rho_{\mathrm{LR}}\right|_{\Pi_{\mathrm{c}}^{\perp}=\hat{\Pi}_{\mathrm{c}}^{\perp}}
$$

Since we are interested in the performance of the filters, we would like to obtain the theoretical behavior of the SINR losses. Some asymptotic studies on the SINR loss in LR Gaussian context have already been done [14], [16]. In [14], [16], the theoretical result is derived by using the assumption that the steering vector is orthogonal to the LR noise and, in this case, [16] obtained an approximation of the expectation of the SINR loss $\hat{\rho}_{\mathrm{LR}}$. However, this assumption is not always verified, not highly relevant and is a restrictive hypothesis in real cases. We consequently propose to relax it and study the convergence of the SINR loss using RMT tools through the study of the nominators and denominators. Indeed, one can already note that the numerators are simple QFs whose convergences were widely considered in RMT. However, the denominators contain more elaborated QFs which were not tackled in RMT in LR context yet and will be the object of Sec.IV.

\section{RANDOM MATRIX THEORY TOOLS}

This section is dedicated to the introduction of classical results from the RMT for the study of the convergence of QFs. This theory and the convergences are based on the behavior of the eigenvalues of the SCM when $m, K \rightarrow \infty$ at the same rate, i.e. $m / K \rightarrow c \in] 0,+\infty)$. In order to simplify the notations, we will abusively note $c=m / K$.

The useful tools for the study of the eigenvalues behavior and the assumptions to the different convergences will be first presented. Secondly, the section will expose the data model, the spiked model [22]. Finally, the useful convergences of simple QFs $\left(\boldsymbol{s}_{1}^{H} \hat{\mathbf{R}}^{-1} \boldsymbol{s}_{2}, \boldsymbol{s}_{1}^{H} \hat{\boldsymbol{\Pi}} \boldsymbol{s}_{2}\right)$ will be introduced.

\section{A. Preliminaries}

The asymptotic behavior of the eigenvalues when $m, K \rightarrow \infty$ at the same rate is described through the convergence of their associated empirical Cumulative Distribution Function $(\mathrm{CDF}) \hat{F}_{m}(x)$ or their empirical Probability Density Function (PDF) $\hat{f}_{m}(x)^{1}$. The asymptotic PDF $f_{m}(x)$ will allow us to characterize the studied data model. The empirical $\mathrm{CDF}$ of the sample eigenvalues of $\hat{\mathbf{R}}$ can be defined as:

$$
\hat{F}_{m}(x)=\frac{1}{m} \#\left\{k: \hat{\lambda}_{k} \leqslant x\right\}
$$

However, in practice, the asymptotic characterization of $\hat{F}_{m}(x)$ is too hard. Consequently, one prefers to study the convergence of the Stieltjes transform $(\mathcal{S T}[\cdot])$ of $\hat{F}_{m}(x)$ :

$$
\begin{aligned}
\hat{b}_{m}(z) & =\mathcal{S T}\left[\hat{F}_{m}(x)\right]=\int_{\mathbb{R}} \frac{1}{x-z} d \hat{F}_{m}(x) \\
& =\frac{1}{m} \sum_{i=1}^{m} \frac{1}{\hat{\lambda}_{i}-z}=\frac{1}{m} \operatorname{tr}\left[\left(\hat{\mathbf{R}}-z \mathbf{I}_{m}\right)^{-1}\right]
\end{aligned}
$$

with $z \in \mathbb{C}^{+} \equiv\{z \in \mathbb{C}: \Im[z]>0\}$ and which almost surely converges to $\bar{b}_{m}(z)$. It is interesting to note that the PDF can thus be retrieve from the Stieltjes transform of its CDF:

$$
\hat{f}_{m}(x)=\lim _{\Im[z] \rightarrow 0} \frac{1}{\pi} \Im\left[\hat{b}_{m}(z)\right]
$$

with $x \in \mathbb{R}$. In other words, the characterization of $\hat{f}_{m}(x)$ (resp. $\left.f_{m}(x)\right)$ can be obtained from $\hat{b}_{m}(z)$ (resp. $\left.\bar{b}_{m}(z)\right)$. Then, to prove the convergences, we assume the following standard hypotheses.

(As1) $\mathbf{R}$ has uniformly bounded spectral norm $\forall m \in \mathbb{N}^{*}$, i.e. $\forall i \in \llbracket 1, m \rrbracket, \lambda_{i}<\infty$.

(As2) The vectors $\boldsymbol{s}_{1}, \boldsymbol{s}_{2} \in \mathbb{C}^{m \times 1}$ used in the QFs (here $\boldsymbol{a}(\boldsymbol{\Theta})$ and $\boldsymbol{x}$ ) have uniformly bounded Euclidean norm $\forall m \in \mathbb{N}^{*}$.

(As3) Let $\mathbf{Y} \in \mathbb{C}^{m \times K}$ having iid entries $y_{i j} \sim \mathcal{C N}(0,1)$. The probability law of $\mathbf{Y}$ is invariant by left multiplication by a deterministic unitary matrix.

\footnotetext{
${ }^{1}$ One can show that under (As1,As3) described later, $\hat{f}_{m}(x)$ a.s. converges towards a nonrandom PDF $f(x)$ with a compact support.
} 
In that case, the following properties are verified. The eigenvalues empirical PDF of $\frac{1}{K} \mathbf{Y} \mathbf{Y}^{H}$ a.s. converges to the MarčenkoPastur distribution [38] with support $\left[(1-\sqrt{c})^{2},(1+\sqrt{c})^{2}\right]$ and the maximum (resp. minimum) eigenvalue of $\frac{1}{K} \mathbf{Y} \mathbf{Y}^{H}$ a.s. tends to $(1+\sqrt{c})^{2}$ (resp. to $\left.(1-\sqrt{c})^{2}\right)$.

\section{B. Covariance matrix models and convergence of eigenvalues}

We first expose the considered data model and, then, the eigenvalues behavior of the SCM. The SCM can be written as $\hat{\mathbf{R}}=\frac{1}{K} \mathbf{X X}^{H}$ with:

$$
\mathbf{X}=\mathbf{R}^{1 / 2} \mathbf{Y}=\left(\mathbf{I}_{m}+\mathbf{P}\right)^{1 / 2} \mathbf{Y}
$$

and $\mathbf{X}=\left[\boldsymbol{x}_{1}, \cdots, \boldsymbol{x}_{K}\right] \cdot \mathbf{R}^{1 / 2}$ is the $m \times m$ Hermitian positive definite square root of the true covariance matrix. The matrix $\mathbf{P}$ is the rank $r$ perturbation matrix and can be eigendecomposed as $\mathbf{P}=\mathbf{U}_{s} \boldsymbol{\Omega} \mathbf{U}_{s}^{H}=\sum_{i=1}^{r} \omega_{i} \mathbf{U}_{i} \mathbf{U}_{i}^{H}$ with:

$$
\boldsymbol{\Omega}=\left[\begin{array}{lll}
\omega_{1} \mathbf{I}_{\mathcal{K}_{1}} & & \\
& \ddots & \\
& & \omega_{r} \mathbf{I}_{\mathcal{K}_{r}}
\end{array}\right]
$$

where $\mathbf{U}_{s}=\left[\mathbf{U}_{1} \cdots \mathbf{U}_{r}\right]$ and $r+1$ is the number of distinct eigenvalues of $\mathbf{R}$. Moreover, $\mathbf{U}_{i} \in \mathbb{C}^{m \times \mathcal{K}_{i}}$ where $\mathcal{K}_{i}$ is the multiplicity of $\omega_{i} . \mathcal{K}_{i}$ is fixed $\forall i \in \llbracket 1, r \rrbracket$ and does not increase with $m$, i.e. $\mathcal{K}_{i} / m_{m, K \rightarrow \infty} 0^{+}, \forall i \in \llbracket 1, r \rrbracket$. For simplicity and in order to fit with our data model, $\mathcal{K}_{i}$ will be set to 1 for all $i \in \llbracket 1, r \rrbracket$. Consequently, we have $\operatorname{rank}(\boldsymbol{\Omega})=\sum_{i \in \llbracket 1, r \rrbracket} \mathcal{K}_{i}=r$ and $\mathcal{K}_{r+1}=m-r$.

Hence, the covariance matrix in Eq.(3) can be rewritten as:

$$
\mathbf{R}=\sum_{i=1}^{r+1} \lambda_{i} \mathbf{U}_{i} \mathbf{U}_{i}^{H}=\mathbf{U}_{r+1} \mathbf{U}_{r+1}^{H}+\sum_{i=1}^{r} \lambda_{i} \boldsymbol{u}_{i} \boldsymbol{u}_{i}^{H}
$$

where $\lambda_{i}$ and $\mathbf{U}_{i}$ are the eigenvalues and the associated subspaces of $\mathbf{R}$ respectively, with $\lambda_{1}=1+\omega_{1}>\cdots>$ $\lambda_{r}=1+\omega_{r}>\lambda_{r+1}=1$ and $\mathbf{U}_{r+1}=\left[\boldsymbol{u}_{r+1} \cdots \boldsymbol{u}_{m}\right]$. In other words, the model specifies that only a few eigenvalues of the LR noise component $c$ are non-unit (and do not contribute to the noise $\boldsymbol{b}$ unit-eigenvalues associated to $\lambda_{r+1}$ ) and fixed.

This model leads to a specific asymptotic eigenvalues PDF of $\mathbf{R}$ as detailed hereafter. The convergence of the eigenvalues is addressed through the convergence of the Stieltjes transform of the eigenvalues CDF. The asymptotic eigenvalue behavior of $\hat{\mathbf{R}}$ for the spiked model was introduced by Johnstone [29] and its eigenvalue behavior was studied in [39]. In order to derive it, [39] exploited the specific expression given in Eq.(20). Then, [22] introduced the final assumption (separation condition) under which the following convergences are given.

(As4.S) The eigenvalues of $\mathbf{P}$ satisfy the separation condition, i.e. $\left|\omega_{i}\right|>\sqrt{c}$ for all $i \in \llbracket 1, r \rrbracket$.

Thus, under (As1-As3, As4.S), we have:

$$
\hat{f}_{m}(x) \underset{\substack{m, K \rightarrow \infty \\ m / K \rightarrow c<\infty}}{\longrightarrow} f(x)
$$

where $f(x)$ is the Marčenko-Pastur law:

$$
f(x)= \begin{cases}\left(1-\frac{1}{c}\right), & \text { if } x=0 \text { and } c>1 \\ \frac{1}{2 \pi c x} \sqrt{\left(\lambda_{-}-x\right)\left(x-\lambda_{+}\right)}, \\ 0, & \text { if } x \in] \lambda_{-}, \lambda_{+}[ \\ \text {otherwise }\end{cases}
$$

with $\lambda_{-}=(1-\sqrt{c})^{2}$ and $\lambda_{+}=(1+\sqrt{c})^{2}$. However, it is essential to note that, for all $i \in \llbracket 1, r \rrbracket$ :

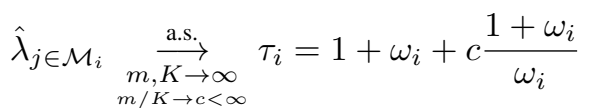

where $\mathcal{M}_{i}$ is the set indexes corresponding to the $j$-th eigenvalue of $\mathbf{R}$ (for example $\mathcal{M}_{r+1}=\{r+1, \cdots, m\}$ for $\left.\lambda_{r+1}\right)$. Two representations of $\hat{f}_{m}(x)$ for two different $c$ and a sufficient large $m$ are shown on Fig. 1 when the eigenvalues of $\mathbf{R}$ are 1,2,3, and 7. The eigenvalues have the same multiplicity and the eigenvalue 1 corresponds the noise eigenvalue. One can observe that (As4.S) is verified if and only if $\tau_{r}>\lambda_{+}$. In other words, all the sample eigenvalues corresponding to the non-unit eigenvalues of $\mathbf{R}$ converge to a value $\tau_{i}$ which is outside the support of the Marčenko-Pastur law ("asymptotic" PDF of the "unit" sample eigenvalues). As an illustration, one can notice that, in Fig. 1, for $\hat{f}_{m}(x)$ plotted for $c=0.1$, the separation condition is verified $\left(\omega_{1}=6\right.$, $\omega_{2}=2$ and $\omega_{3}=1$ are greater than $\left.\sqrt{c}=0.316\right)$ and the three non-unit eigenvalues are represented on the PDF and outside the support of the Marčenko-Pastur law by their respective limits $\tau_{1}=7.116, \tau_{2}=3.15$ and $\tau_{3}=2.2$. On the contrary, for $\hat{f}_{m}(x)$ plotted for $c=1.5$, only the two greatest eigenvalues are represented on the PDF by their respective limits $\tau_{1}=8.75$ and $\tau_{2}=5.25$ while the separation condition is not verified for the eigenvalue $\lambda_{3}=2\left(\omega_{3}=1<\sqrt{c}=1.223\right)$. In this case, the sample eigenvalues corresponding to the eigenvalue $\lambda_{3}=2$ belongs to the Marčenko-Pastur law.

\section{Convergence of simple quadratic forms}

Here, we compare the convergence of two simple QFs in two convergence regimes: when $K \rightarrow \infty$ with a fixed $m$ and when $m, K \rightarrow \infty$ at the same rate.

We first present the useful convergences of simple QFs function of $\hat{\mathbf{R}}$. It is well known that, due to the strong law of large numbers, when $K \rightarrow \infty$ with a fixed $m, \hat{\mathbf{R}} \rightarrow \mathbf{R}$ a.s. [40]. Thus,

$$
\boldsymbol{s}_{1}^{H} \hat{\mathbf{R}}^{-1} \boldsymbol{s}_{2} \underset{\substack{\mathrm{K} \rightarrow \infty \\ \mathrm{m} \rightarrow \infty}}{\stackrel{\text { a.s. }}{\longrightarrow}} \boldsymbol{s}_{1}^{H} \mathbf{R}^{-1} \boldsymbol{s}_{2}
$$

Moreover, when $m, K \rightarrow \infty$ at the same rate [19], [41]:

$$
\boldsymbol{s}_{1}^{H} \hat{\mathbf{R}}^{-1} \boldsymbol{s}_{2} \underset{\substack{m, K \rightarrow \infty \\ m / K \rightarrow \infty}}{\stackrel{\text { a.s. }}{\longrightarrow}}(1-c)^{-1} \boldsymbol{s}_{1}^{H} \mathbf{R}^{-1} \boldsymbol{s}_{2}
$$

The useful convergences of simple QFs function of $\hat{\boldsymbol{\Pi}}_{\mathrm{c}}^{\perp}$ are hereafter presented. As $\hat{\mathbf{R}} \rightarrow \mathbf{R}$ a.s. when $K \rightarrow \infty$ with a fixed $m, \hat{\boldsymbol{\Pi}}_{\mathrm{c}}^{\perp} \rightarrow \boldsymbol{\Pi}_{\mathrm{c}}^{\perp}$ a.s. [19] in the same convergence regime. Thus:

$$
\boldsymbol{s}_{1}^{H} \hat{\boldsymbol{\Pi}}_{\mathrm{c}}^{\perp} \boldsymbol{s}_{2} \underset{\substack{\mathrm{K} \rightarrow \infty \\ \mathrm{K} \rightarrow \infty}}{\stackrel{\text { a.s. }}{\longrightarrow}} \boldsymbol{s}_{1}^{H} \boldsymbol{\Pi}_{\mathrm{c}}^{\perp} \boldsymbol{s}_{2}
$$




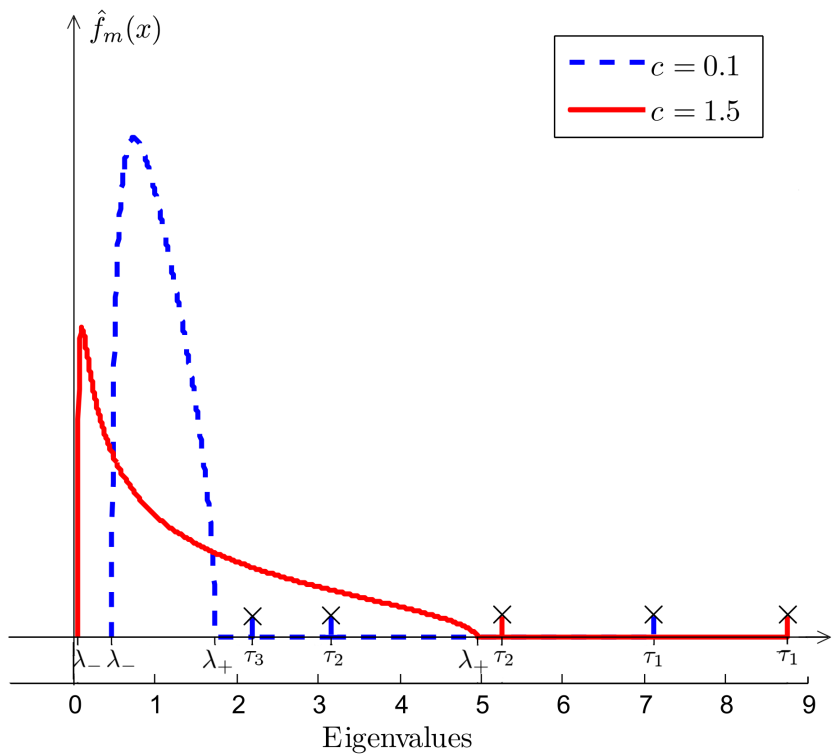

Fig. 1. PDF of the eigenvalues of the SCM with the spiked model when the eigenvalues of $\mathbf{R}$ are 1,2,3, and 7 with the same multiplicity, where 1 is the noise eigenvalue.

For the convergences in the large dimensional regime ( $m, K \rightarrow \infty$ at the same rate), they are presented under (As1As3) and the separation condition As4.S. [22] showed that, $\forall i \in \llbracket 1, r \rrbracket$ :

$$
\boldsymbol{s}_{1}^{H} \hat{\mathbf{U}}_{i} \hat{\mathbf{U}}_{i}^{H} \boldsymbol{s}_{2} \underset{\substack{m, K \rightarrow \infty<\infty \\ m / K \rightarrow c<\infty}}{\stackrel{\text { a.s. }}{\longrightarrow}} \frac{1-c \omega_{i}^{-2}}{1+c \omega_{i}^{-1}} \boldsymbol{s}_{1}^{H} \mathbf{U}_{i} \mathbf{U}_{i}^{H} \boldsymbol{s}_{2}
$$

where $\omega_{i}=\lambda_{i}-1$ and $\lambda_{i}$ is the $i$-th distinct eigenvalue of $\mathbf{R}$. Let $\chi_{i}=\frac{1-c \omega_{i}^{-2}}{1+c \omega_{i}^{-1}}$. Thus, using the following relationship,

$$
\hat{\boldsymbol{\Pi}}_{\mathrm{c}}^{\perp}=\mathbf{I}_{m}-\sum_{i=1}^{r} \hat{\boldsymbol{u}}_{i} \hat{\boldsymbol{u}}_{i}^{H}
$$

one can deduce that with the spiked model and in the large dimensional regime:

$$
\boldsymbol{s}_{1}^{H} \hat{\boldsymbol{\Pi}}_{\mathrm{c}}^{\perp} \boldsymbol{s}_{2} \underset{\substack{m, K \rightarrow K \rightarrow \infty \\ m / K \rightarrow \infty<\infty}}{\stackrel{\text { a.s. }}{\longrightarrow}} \boldsymbol{s}_{1}^{H} \overline{\boldsymbol{\Pi}}_{\mathrm{c}, \mathrm{S}}^{\perp} \boldsymbol{s}_{2}
$$

with $\overline{\boldsymbol{\Pi}}_{\mathrm{c}, \mathrm{S}}^{\perp}=\sum_{i=1}^{m} \psi_{i} \boldsymbol{u}_{i} \boldsymbol{u}_{i}^{H}$ and

$$
\psi_{i}= \begin{cases}1, & \text { if } i>r \\ 1-\chi_{i}, & \text { if } i \leqslant r\end{cases}
$$

To summarize, $\boldsymbol{s}_{1}^{H} \hat{\mathbf{R}}^{-1} \boldsymbol{s}_{2}$ is consistent when $K \rightarrow \infty$ with a fixed $m$ and its limits in the large dimensional regime is proportional to the theoretical $\mathrm{QF} s_{1}^{H} \mathbf{R}^{-1} s_{2}$. However, although $\boldsymbol{s}_{1}^{H} \hat{\boldsymbol{\Pi}}_{\mathrm{c}}^{\perp} \boldsymbol{s}_{2}$ is consistent when $K \rightarrow \infty$ with a fixed $m$, it is no more consistent under the regime of interest i.e. when both $m, K \rightarrow \infty$ at the same rate. More precisely, the limit of $\boldsymbol{s}_{1}^{H} \hat{\boldsymbol{\Pi}}_{\mathrm{c}}^{\perp} \boldsymbol{s}_{2}$ in the large dimensional regime is not purely proportional to the theoretical QF $s_{1}^{H} \Pi_{\mathrm{c}}^{\perp} s_{2}$ but a completely different QF.

\section{NEW CONVERGENCE RESUlTS}

\section{A. Convergence of LR structured quadratic forms}

In this section, the convergence of the structured $\mathrm{QF}$ function of $\hat{\boldsymbol{\Pi}}_{\mathrm{c}}^{\perp}$ is analyzed and results in Proposition 1.

Proposition 1: Let $\mathbf{B}$ be a $m \times m$ deterministic complex matrix with a uniformly bounded spectral norm for all $m$. Then, under (As1-As3, As4.S) and the spiked model,

$$
\boldsymbol{s}_{1}^{H} \hat{\boldsymbol{\Pi}}_{\mathrm{c}}^{\perp} \mathbf{B} \hat{\boldsymbol{\Pi}}_{\mathrm{c}}^{\perp} \boldsymbol{s}_{2} \underset{\substack{m, K \rightarrow \infty \\ m / K \rightarrow c<\infty}}{\stackrel{\text { a.s. }}{\longrightarrow}} \boldsymbol{s}_{1}^{H} \overline{\boldsymbol{\Pi}}_{\mathrm{c}, \mathrm{S}}^{\perp} \mathbf{B} \overline{\boldsymbol{\Pi}}_{\mathrm{c}, \mathrm{S}}^{\perp} \boldsymbol{s}_{2}
$$

where $\overline{\boldsymbol{\Pi}}_{\mathrm{c}, \mathrm{S}}^{\perp}=\sum_{i=1}^{m} \psi_{i} \boldsymbol{u}_{i} \boldsymbol{u}_{i}^{H}$ with $\psi_{i}$ defined by Eq.(32).

\section{Proof: See Appendix.}

Moreover, one can remark that if $\mathbf{B}=\mathbf{R}$, where $\mathbf{R}$ is the covariance matrix as defined in Eq.(5), the following convergence holds:

$$
\boldsymbol{s}_{1}^{H} \hat{\boldsymbol{\Pi}}_{\mathrm{c}}^{\perp} \mathbf{R} \hat{\boldsymbol{\Pi}}_{\mathrm{c}}^{\perp} \boldsymbol{s}_{2} \underset{\substack{m, K \rightarrow \infty \\ m / K \rightarrow c<\infty}}{\stackrel{\text { a.s. }}{\longrightarrow}} \boldsymbol{s}_{1}^{H} \overline{\boldsymbol{\Pi}}_{\mathrm{c}, \mathrm{S}}^{\perp} \mathbf{R} \overline{\boldsymbol{\Pi}}_{\mathrm{c}, \mathrm{S}}^{\perp} \boldsymbol{s}_{2}
$$

A visualization of the convergence of Eq.(33) in terms of Mean Squared Error (MSE) can be found in Fig. 2 when $m, K \rightarrow \infty$ at a fixed ratio. It is compared to the MSE corresponding to the following convergence when $K \rightarrow \infty$ with a fixed $m$ :

$$
\boldsymbol{s}_{1}^{H} \hat{\boldsymbol{\Pi}}_{\mathrm{c}}^{\perp} \mathbf{B} \hat{\Pi}_{\mathrm{c}}^{\perp} \boldsymbol{s}_{2} \underset{\substack{\mathrm{K} \rightarrow \infty \\ m<\infty}}{\text { a.s. }} \boldsymbol{s}_{1}^{H} \boldsymbol{\Pi}_{\mathrm{c}}^{\perp} \mathbf{B} \boldsymbol{\Pi}_{\mathrm{c}}^{\perp} \boldsymbol{s}_{2}
$$

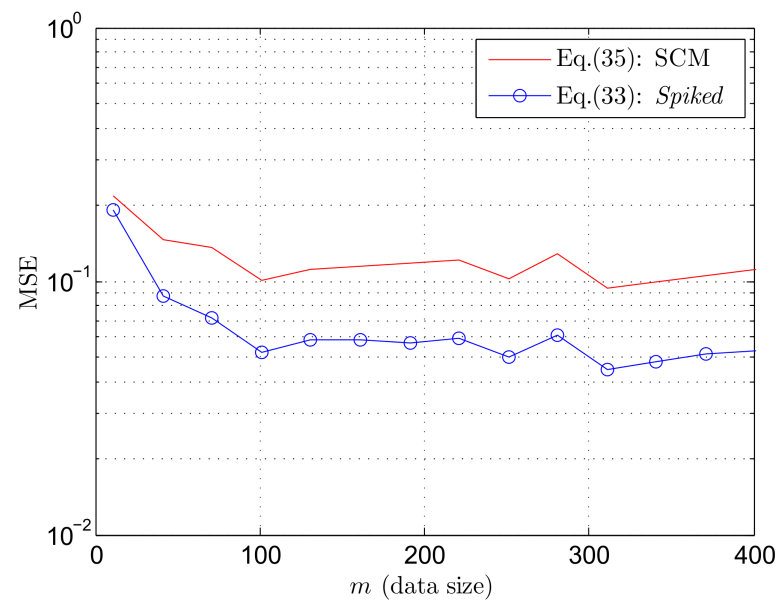

Fig. 2. MSE over $10^{3}$ iterations corresponding to Eq.(33) and Eq.(35) when the eigenvalues of $\mathbf{R}$ are $1,21,31$, and 71 with the multiplicity $m-3,1$, 1 and 1 respectively, $c=0.1, s_{1}=s_{2}$ are steering vectors of the LR noise component $\boldsymbol{c}$ and $\mathbf{B}=\mathbf{R}$

\section{B. Convergence of SINR losses}

Now, we provide the convergences of the estimated SINR losses using the convergences previously presented and the following convergence. We recall that, as $\hat{\mathbf{R}} \rightarrow \mathbf{R}$ a.s. when $K \rightarrow \infty$ with a fixed $m$, one has:

$$
\boldsymbol{s}_{1}^{H} \hat{\mathbf{R}}^{-1} \mathbf{R} \hat{\mathbf{R}}^{-1} \boldsymbol{s}_{2} \underset{\substack{\text { a.s. } \\ m<\infty}}{\longrightarrow} s_{1}^{H} \mathbf{R}^{-1} \boldsymbol{s}_{2}
$$


Hence, when $K \rightarrow \infty$ with a fixed $m$ and using Eq.(26), Eq.(36) and the continuous mapping theorem [40]:

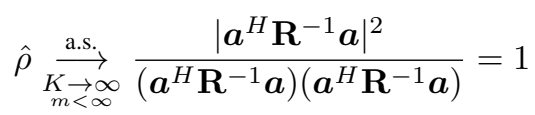

And, under (As1-As3), when $m, K \rightarrow \infty$ at the same rate, from [33], [35], [36], we have:

$$
\hat{\rho} \underset{\substack{m, K \rightarrow \infty \\ m / K \rightarrow c<\infty}}{\stackrel{\text { a.s. }}{\longrightarrow}} \frac{(1-c)\left|\boldsymbol{a}^{H} \mathbf{R}^{-1} \boldsymbol{a}\right|^{2}}{\left(\boldsymbol{a}^{H} \mathbf{R}^{-1} \boldsymbol{a}\right)\left(\boldsymbol{a}^{H} \mathbf{R}^{-1} \boldsymbol{a}\right)}=1-c
$$

To summarize, $\boldsymbol{s}_{1}^{H} \hat{\mathbf{R}}^{-1} \boldsymbol{s}_{2}$ is consistent when $K \rightarrow \infty$ with a fixed $m$ and its limits in the large dimensional regime is proportional to the theoretical QF $s_{1}^{H} \mathbf{R}^{-1} s_{2}$. Thus, the estimated SINR loss $\hat{\rho}$ is consistent when $K \rightarrow \infty$ with $m$ fixed and its limits in the large dimensional regime is proportional to $\rho$. Consequently, RMT cannot help us to improve the estimation of the theoretical SINR loss as a function of $r$.

For the SINR loss corresponding to the adaptive LR filters, when $K \rightarrow \infty$ with a fixed $m$, using Eq.(28), Eq.(35) and the continuous mapping theorem, we have:

$$
\hat{\rho}_{\mathrm{LR}} \underset{\substack{K \rightarrow \infty \\ m<\infty}}{\stackrel{\text { a.s. }}{\longrightarrow}} \rho_{\mathrm{LR}}
$$

where $\rho_{\mathrm{LR}}$ is defined by Eq.(14). When $m, K \rightarrow \infty$ at the same rate, we obtain the following convergence:

$$
\hat{\rho}_{\mathrm{LR}} \underset{\substack{m, K \rightarrow \infty \\ m / K \rightarrow c<\infty}}{\stackrel{\text { a.s. }}{\longrightarrow}} \bar{\rho}_{\mathrm{LR}}^{(\mathrm{S})}=\left.\rho_{\mathrm{LR}}\right|_{\boldsymbol{\Pi}_{\mathrm{c}}^{\perp}=\overline{\boldsymbol{\Pi}}_{\mathrm{c}, \mathrm{S}}^{\perp}} \neq \rho_{\mathrm{LR}}
$$

where Eq.(31), Proposition 1 and the continuous mapping theorem were used to prove Eq.(40). One can observe that, although the traditional estimator of $\rho_{\mathrm{LR}}$ is consistent when $K \rightarrow \infty$ with a fixed $m$, it is no more consistent when $m, K \rightarrow \infty$ at the same rate. It is also important to underline that the new convergence result leads to a more precise approximation of $\hat{\rho}_{\mathrm{LR}}$ than previous works [14], [16]. Indeed, [14], [16] propose an approximation dependent on $K$ and $r$. In this article, the proposed approximation depends on $K$ (and of course on $c$ ) and $r$ as well as on the parameter $\Theta$, which allows us to have a performance measure function of the "distance" between the signal of interest and the jamming (typically $\left.\left\{\boldsymbol{u}_{i}^{H} \boldsymbol{a}(\boldsymbol{\Theta})=g_{i}\left(\boldsymbol{\Theta}_{\text {jamming }}, \boldsymbol{\Theta}\right)\right\}_{i \in \llbracket 1, r \rrbracket} \in[-1,1]\right)$.

\section{Simulations}

\section{A. Parameters}

As an illustration of the interest of the RMT utilization in filtering, the jamming application is chosen. The purpose of this application is to detect a target thanks to a ULA composed of $m$ sensors despite the presence of jamming. The response of the jamming, $c$ is composed of signals similar to the target response. In this section, except for the convergences when $m, K \rightarrow \infty$ at the same rate $c$, we choose $m=100$ in order to have a large number for the data dimension. Even if, in some basic array processing applications, this number could seem significant, it actually became standard in many applications such as STAP [2], MIMO applications [42], [43],
MIMO-STAP [42], etc. Here, $\Theta=\theta$ where $\theta$ is the AoA. The jamming is composed of three synthetic targets with AoA $-20^{\circ}, 0^{\circ}$ and $20^{\circ}$ and wavelength $l_{0}=0.667 \mathrm{~m}$. Thus, the jamming (LR noise) has a rank $r=3$. Then, the AWGN $\boldsymbol{b}$ power is $\sigma^{2}=1$. Finally, the theoretical covariance matrix of the total noise can be written as $\mathbf{R}=\frac{J N R}{\operatorname{tr}(\boldsymbol{\Lambda})} \mathbf{U} \boldsymbol{\Lambda} \mathbf{U}^{H}+\sigma^{2} \mathbf{I}_{m}$ with $\boldsymbol{\Lambda}=\operatorname{diag}([6,2,1])$ and where $J N R$ is the jamming to noise ratio. $\frac{J N R}{\operatorname{tr}(\boldsymbol{\Lambda})}$ is set at $10 \mathrm{~dB}$ except for Fig. 4 .

In order to validate the spiked model as covariance matrix model, we visualize a zoom of the experimental PDF of the eigenvalues of our data without target in Fig. 3 over $5 \times 10^{4}$ Monte-Carlo iterations. We observe a Marčenko-Pastur law around 1 (eigenvalues of the white noise) and Gaussian distributions for the eigenvalues of the jamming, which is consistent to the CLT for the spiked model proved in [22]. The spiked model is consequently relevant for our data model.

Moreover, in order to verify that the spiked model

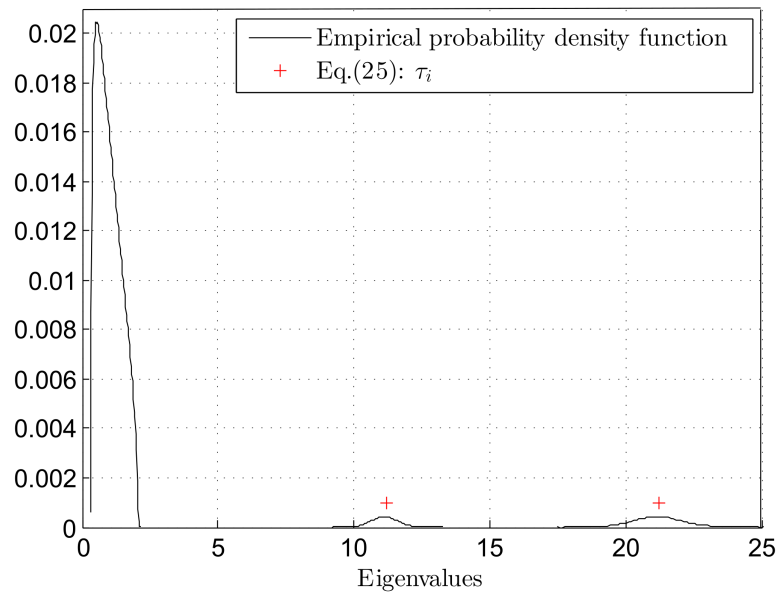

Fig. 3. Zoom of the experimental PDF of jamming plus noise data with $c=0.2$ and $\frac{J N R}{\operatorname{tr}(\boldsymbol{\Lambda})}=10 \mathrm{~dB}$. Due to the zoom, the highest eigenvalue 71 is out of the figure.

is realistic in terms of separation condition, Fig. 4 shows $\left(\omega_{r}-\sqrt{c}\right)$ as a function of $\frac{J N R}{\operatorname{tr}(\boldsymbol{\Lambda})}$ in $\mathrm{dB}$. We recall that, in order to satisfy the separation condition, one should have $\omega_{r}-\sqrt{c}>0$. Consequently, we gladly observe that it is satisfied for $\frac{J N R}{\operatorname{tr}(\boldsymbol{\Lambda})}>6.2 \mathrm{~dB}$ for the majority of $c$ even if $c>17$. Indeed, in practice, if the $\frac{J N R}{\operatorname{tr}(\boldsymbol{\Lambda})}$ is lower than $6.2 \mathrm{~dB}$, the jamming will not have any effects on the performance. Consequently, the chosen parameters are realistic even if $K=2 r$ (implying $c \simeq 16.7$ ).

\section{B. Performance of filters}

We now observe the performance of filters through the SINR loss. We are first interested in the validation of the convergence of $\hat{\rho}_{\mathrm{LR}}$ in Eq.(40) as $m, K \rightarrow \infty$ at the same rate. This convergence is validated and presented in Fig. 5 in terms of MSE over $10^{3}$ realizations with $c=3$ for an AoA of the target $\left(\theta=50^{\circ}\right)$ and an AoA of the jamming $\left(\theta=20^{\circ}\right)$.

Fig. 6 shows the visualization of Eq.(14) (blue line with stars), Eq.(15) (blue dashed line), the right side of the convergence in Eq.(40) (green line with circles) and the approximation $\mathbb{E}\left[\hat{\rho}_{\mathrm{LR}}\right] \simeq 1-\frac{r}{K}$ introduced by [14] (black 


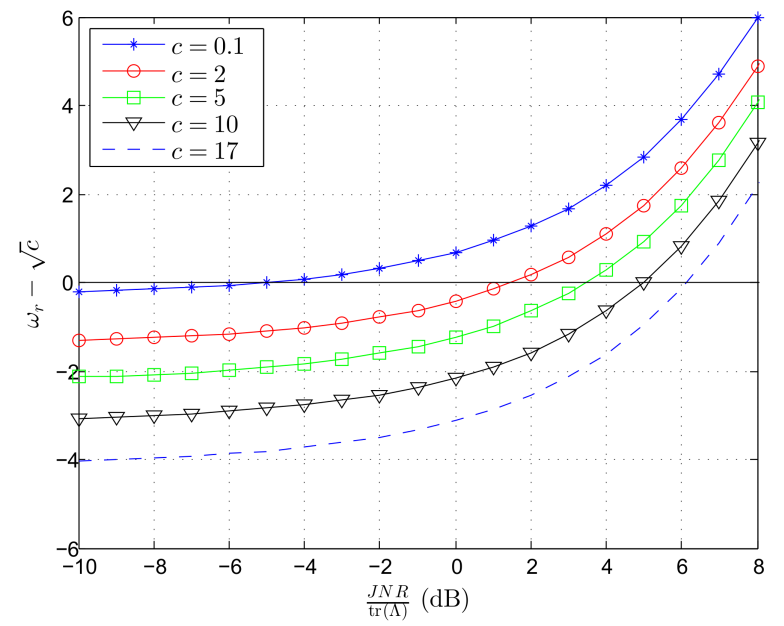

Fig. 4. Separation condition $\left(\omega_{r}-\sqrt{c}\right)$ of the spiked model for the lowest non-unit eigenvalue as a function of the ratio $\frac{J N R}{\operatorname{tr}(\Lambda)}$ in $\mathrm{dB}$.

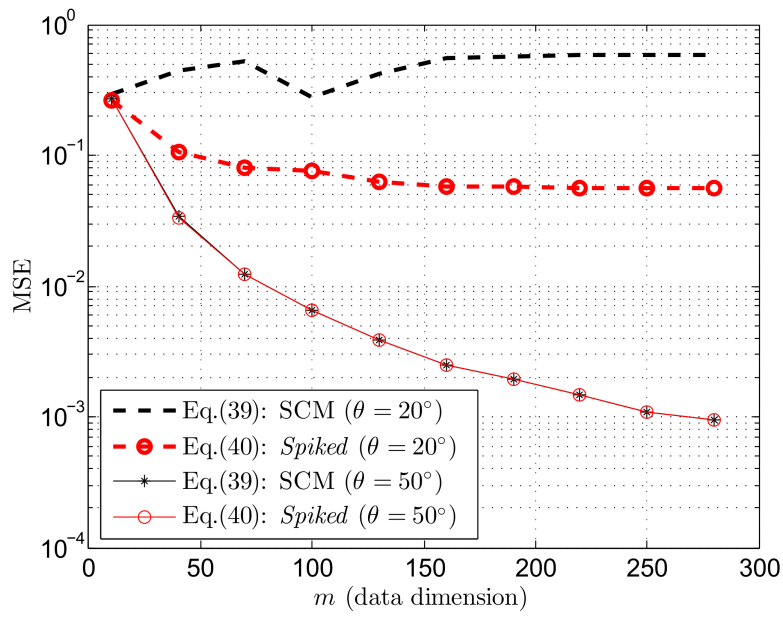

Fig. 5. MSE corresponding to Eq.(39) and Eq.(40) when $m, K \rightarrow \infty$ at a fixed ratio $c=3$ and $\frac{J N R}{\operatorname{tr}(\boldsymbol{\Lambda})}=10 \mathrm{~dB}$.

line) as a function of $K$ when the target is near from the jamming, i.e. $\theta=20.5^{\circ}$. We observe that the spiked model and the RMT helps us to obtain a better estimation of $\mathbb{E}\left[\hat{\rho}_{\mathrm{LR}}\right]$ than the estimation $\mathbb{E}\left[\hat{\rho}_{\mathrm{LR}}\right] \simeq 1-\frac{r}{K}$ as the curve of $\bar{\rho}_{\mathrm{LR}}^{(\mathrm{S})}$ has the same behavior as the curve of $\hat{\rho}_{\mathrm{LR}}$. Then, similarly, the same equations are visualized as a function of $\theta$ in Fig. 7 with $K=2 r$. We observe that, unlike the estimation $1-r / K$, the RMT with the spiked model permits us to obtain a better estimation of $\mathbb{E}\left[\hat{\rho}_{\mathrm{LR}}\right]$ as a function of $\theta$ and consequently a better approximation of its behavior. Thus, it permits to predict the parameter $\theta$ value corresponding to the performance break (here around $21.1^{\circ}$ ).

\section{CONCLUSION}

In this paper, we proposed new results in random matrix theory with a specific covariance matrix model fitted to our data model: the spiked model. Based on this, we studied the convergence of the traditional estimator of the SINR loss in its low rank version when the number of secondary data $K \rightarrow \infty$ with a fixed data dimension $m$ and when $m, K \rightarrow \infty$ at

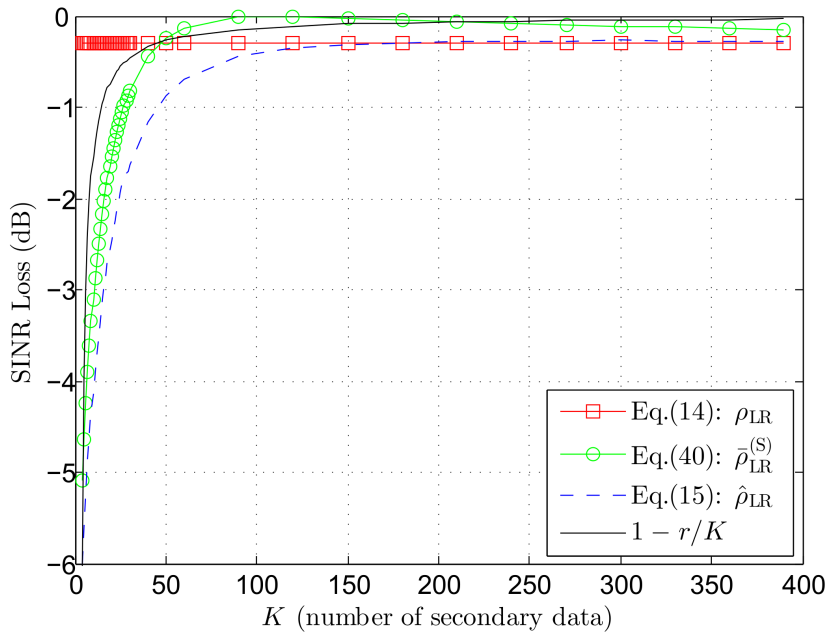

Fig. 6. Visualization of Eq.(14) (red line with squares), Eq.(15) (blue dashed line), the right side of the convergence in Eq.(40) (green line with circles) and the traditional estimation of $\mathbb{E}\left[\hat{\rho}_{\mathrm{LR}}\right]$ (black line) as a function of $K$ (over $10^{3}$ realizations) with $\frac{J N R}{\operatorname{tr}(\boldsymbol{\Lambda})}=10 \mathrm{~dB}, m=100$ and $\theta=20.5^{\circ}$.

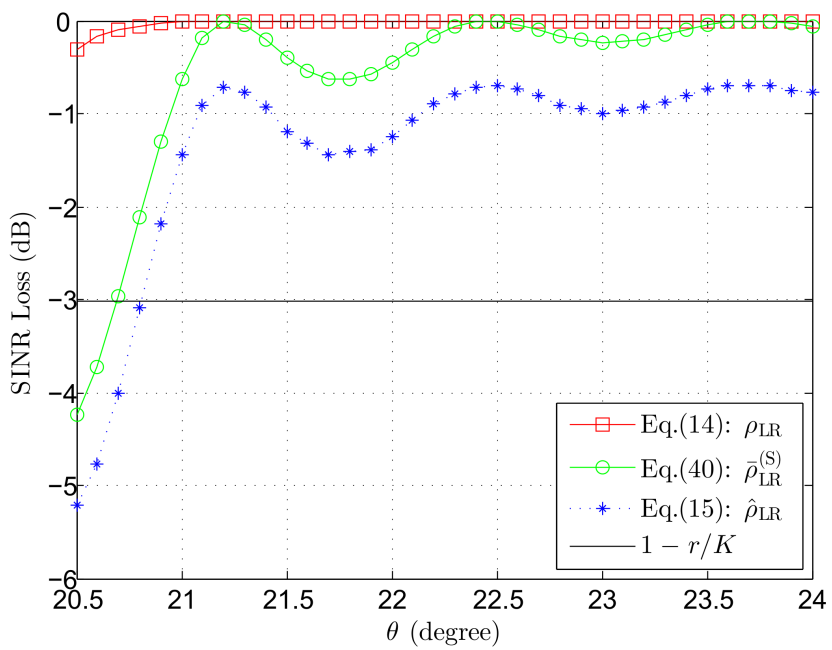

Fig. 7. Visualization of Eq.(14) (red line with squares), Eq.(15) (blue dashed line), the right side of the convergence in Eq.(40) (green line with circles) and the traditional estimation of $\mathbb{E}\left[\hat{\rho}_{\mathrm{LR}}\right]$ (black line) as a function of $\theta$ (over $10^{3}$ realizations) with $\frac{J N R}{\operatorname{tr}(\boldsymbol{\Lambda})}=10 \mathrm{~dB}, m=100$ and $K=2 r$.

the same rate $c=m / K$ as random matrix theory cannot help us with its full rank version to obtain performance function of $c$ and the distance between the target and the jammers. We observed the low rank version is consistent when $K \rightarrow \infty$ with a fixed $m$ but is not consistent when $m, K \rightarrow \infty$ at the same rate $c$. Finally, we applied these results to a jamming application. We first observed that the experimental probability density function of the eigenvalues of the covariance matrix of jamming data is relevant with the probability density function of the eigenvalues corresponding to the spiked model. Then, we validated the convergence of the SINR loss in its low rank version. We observed that random matrix theory and more precisely the spiked model better evaluate the asymptotic performance of the low rank SINR loss corresponding to the adaptive low rank filter, especially when the steering vector parameter is close to the jamming 
one. Thus, contrary to previous works, it allows us to predict the filter performance when the signal of interest is close to the low rank noise. Consequently, this new result permits to predict the steering vector parameter value corresponding to the performance break.

\section{APPENDIX}

The proof is decomposed as follows. We first develop the structured $\mathrm{QF}$ as a sum of simple QFs and base structured QF (Subsec. VII-A). In a second time, we formulate the base structured QF as a complex integral (Subsec. VII-B) and split it into several integrals (Subsec. VII-C). Then, we determine the deterministic complex integral equivalent of the base structured QF (Subsec. VII-D) and its formal expression (Subsec. VII-E). Finally, we use this result to determine the convergence of the structured $\mathrm{QF}$ in the large dimensional regime (Subsec. VII-F). The regime of convergences in the Appendix, if not precised, is $m, K \rightarrow \infty$ at a fixed ratio $c$.

\section{A. Development of the structured $Q F$}

Let $s_{1}$ and $s_{2}$ be two deterministic complex vectors and B be a $m \times m$ deterministic complex matrix with uniformly bounded spectral norm for all $\mathrm{m}$. In order to obtain the convergence of the structured $\mathrm{QF} \boldsymbol{s}_{1}^{H} \hat{\boldsymbol{\Pi}}_{\mathrm{c}}^{\perp} \mathrm{B} \hat{\boldsymbol{\Pi}}_{\mathrm{c}}^{\perp} \boldsymbol{s}_{2}$, one can rewrite, using the notations of Eq.(22) and the spiked model, $\hat{\boldsymbol{\Pi}}_{\mathrm{c}}^{\perp}=\hat{\boldsymbol{\Pi}}_{r+1}=\hat{\mathbf{U}}_{r+1} \hat{\mathbf{U}}_{r+1}^{H}=\mathbf{I}_{m}-\sum_{i=1}^{r} \hat{\boldsymbol{\Pi}}_{i}$ where $\hat{\mathbf{U}}_{r+1}=\left[\hat{\boldsymbol{u}}_{r+1}, \cdots, \hat{\boldsymbol{u}}_{m}\right], \hat{\boldsymbol{\Pi}}_{i}=\hat{\boldsymbol{u}}_{i} \hat{\boldsymbol{u}}_{i}^{H}, \forall i \in \llbracket 1, r \rrbracket$ and $\hat{\boldsymbol{u}}_{i}$ are the eigenvectors of the SCM. We recall that $r$ is fixed for all $m$, i.e. $r / m \rightarrow 0^{+}$and the notation $\mathbf{U}_{s}=\left[\boldsymbol{u}_{1}, \cdots, \boldsymbol{u}_{r}\right]$. Thus, one can develop the structured $\mathrm{QF}$ as :

$$
\begin{aligned}
\boldsymbol{s}_{1}^{H} \hat{\boldsymbol{\Pi}}_{\mathrm{c}}^{\perp} \mathbf{B} \hat{\boldsymbol{\Pi}}_{\mathrm{c}}^{\perp} \boldsymbol{s}_{2}= & \boldsymbol{s}_{1}^{H}\left(\mathbf{I}_{m}-\sum_{i=1}^{r} \hat{\boldsymbol{\Pi}}_{i}\right) \mathbf{B}\left(\mathbf{I}_{m}-\sum_{i=1}^{r} \hat{\boldsymbol{\Pi}}_{i}\right) \boldsymbol{s}_{2} \\
= & \boldsymbol{s}_{1}^{H} \mathbf{B} \boldsymbol{s}_{2}-\boldsymbol{s}_{1}^{H} \sum_{i=1}^{r} \hat{\boldsymbol{\Pi}}_{i} \mathbf{B} \boldsymbol{s}_{2}-\boldsymbol{s}_{1}^{H} \mathbf{B} \sum_{i=1}^{r} \hat{\boldsymbol{\Pi}}_{i} \boldsymbol{s}_{2} \\
& \quad+\boldsymbol{s}_{1}^{H} \sum_{i=1}^{r} \hat{\boldsymbol{\Pi}}_{i} \mathbf{B} \sum_{i=1}^{r} \hat{\boldsymbol{\Pi}}_{i} \boldsymbol{s}_{2} \\
= & \boldsymbol{s}_{1}^{H} \mathbf{B} \boldsymbol{s}_{2}-\sum_{i=1}^{r}\left(\boldsymbol{s}_{1}^{H} \hat{\boldsymbol{\Pi}}_{i} \mathbf{B} \boldsymbol{s}_{2}+\boldsymbol{s}_{1}^{H} \mathbf{B} \hat{\boldsymbol{\Pi}}_{i} \boldsymbol{s}_{2}\right) \\
& +\sum_{j_{1}=1}^{r} \boldsymbol{s}_{1}^{H} \hat{\boldsymbol{\Pi}}_{j_{1}} \mathbf{B} \hat{\boldsymbol{\Pi}}_{j_{1}} \boldsymbol{s}_{2}+\sum_{\substack{j_{1}, j_{2}=1 \\
j_{1} \neq j_{2}}}^{r} \boldsymbol{s}_{1}^{H} \hat{\boldsymbol{\Pi}}_{j_{1}} \mathbf{B} \hat{\boldsymbol{\Pi}}_{j_{2}} \boldsymbol{s}_{2}(
\end{aligned}
$$

B. Formulation of the base structured $Q F$ as a complex integral

Remarking that Eq.(43) is a sum of simple QFs and base structured QFs, we first focus on the convergence of the base structured QF $\hat{\eta}\left(j_{1}, j_{2}\right)=\boldsymbol{s}_{1}^{H} \hat{\boldsymbol{\Pi}}_{j_{1}} \mathbf{B} \hat{\boldsymbol{\Pi}}_{j_{2}} \boldsymbol{s}_{2},\left\{j_{1}, j_{2}\right\} \in \llbracket 1, r \rrbracket^{2}$. Let us now formulate the base structured $\mathrm{QF}$ as a complex integral.

Proposition 2: Let B be a $m \times m$ deterministic complex matrix with a uniformly bounded spectral norm for all $m$. Then, under (As1-As3, As4.S) and the spiked model, $\forall j_{1}, j_{2} \in$ $\llbracket 1, r \rrbracket$, if $\hat{\eta}\left(j_{1}, j_{2}\right)=\boldsymbol{s}_{1}^{H} \hat{\boldsymbol{\Pi}}_{j_{1}} \mathbf{B} \hat{\boldsymbol{\Pi}}_{j_{2}} \boldsymbol{s}_{2}$ :

$$
\begin{aligned}
\hat{\eta}\left(j_{1}, j_{2}\right)= & \frac{1}{(2 i \pi)^{2}} \oint_{\mathcal{C}_{j_{1}}^{-}} \oint_{\mathcal{C}_{j_{2}}^{-}} \boldsymbol{s}_{1}^{H}\left(\hat{\mathbf{R}}-z_{1} \mathbf{I}_{m}\right)^{-1} \\
& \times \mathbf{B}\left(\hat{\mathbf{R}}-z_{2} \mathbf{I}_{m}\right)^{-1} \boldsymbol{s}_{2} d z_{1} d z_{2}
\end{aligned}
$$

Proof: If $j_{1} \neq j_{2}$, it can be easily shown that $\hat{\eta}\left(j_{1}, j_{2}\right)$ can be expressed as the following Cauchy integral in two complex variables [44]:

$$
A=\frac{1}{(2 i \pi)^{2}} \oint_{\mathcal{C}_{j_{1}}^{-}} \oint_{\mathcal{C}_{j_{2}}^{-}} \boldsymbol{s}_{1}^{H}\left(\hat{\mathbf{R}}-z_{1} \mathbf{I}_{m}\right)^{-1} \mathbf{B}\left(\hat{\mathbf{R}}-z_{2} \mathbf{I}_{m}\right)^{-1} \boldsymbol{s}_{2} d z_{1} d z_{2}
$$

where $\mathcal{C}_{j}^{-}$in a negatively oriented contour encompassing the eigenvalues of $\hat{\mathbf{R}}$ corresponding to the $j$-th eigenvalue of $\mathbf{R}$ and $z_{1}$ and $z_{2}$ are independent variables.

Then, if $j_{1}=j_{2}=j \in \llbracket 1, r \rrbracket$, one has:

$$
\boldsymbol{s}_{1}^{H} \hat{\boldsymbol{\Pi}}_{j} \mathbf{B} \hat{\boldsymbol{\Pi}}_{j} \boldsymbol{s}_{2}=\frac{1}{2 i \pi} \oint_{\mathcal{C}_{j}^{-}} \boldsymbol{s}_{1}^{H} \sum_{n=1}^{m} \frac{\hat{\boldsymbol{u}}_{n} \hat{\boldsymbol{u}}_{n}^{H} \mathbf{B} \hat{\boldsymbol{u}}_{n} \hat{\boldsymbol{u}}_{n}^{H}}{\hat{\lambda}_{n}-z} \boldsymbol{s}_{2} d z
$$

However, the remaining of the proof is based on the fact that the resolvent $\mathbf{G}(z)$ of the SCM can be found in the complex integral, which is not the case in the previous equation. Consequently, even if Eq.(46) is equivalent to Eq.(44), it will be easier to use Eq.(44). As a consequence, $\forall j_{1}, j_{2} \in \llbracket 1, r \rrbracket$ :

$$
\begin{aligned}
\hat{\eta}\left(j_{1}, j_{2}\right)= & \frac{1}{(2 i \pi)^{2}} \oint_{\mathcal{C}_{j_{1}}^{-}} \oint_{\mathcal{C}_{j_{2}}^{-}} \boldsymbol{s}_{1}^{H}\left(\hat{\mathbf{R}}-z_{1} \mathbf{I}_{m}\right)^{-1} \\
& \times \mathbf{B}\left(\hat{\mathbf{R}}-z_{2} \mathbf{I}_{m}\right)^{-1} \boldsymbol{s}_{2} d z_{1} d z_{2}
\end{aligned}
$$

\section{Development of the complex integral}

Next, one want to split the previous line integral into several line integrals where some of them will tend to 0 . Thus, from [22], with $k \in\{1,2\}$, one can write:

$$
\begin{aligned}
\left(\hat{\mathbf{R}}-z_{k} \mathbf{I}_{m}\right)^{-1}= & \left(\mathbf{I}_{m}+\mathbf{P}\right)^{-1 / 2}\left[\mathbf{Q}\left(z_{k}\right)-z_{k} \mathbf{Q}\left(z_{k}\right) \mathbf{U}_{s}\right. \\
& \left.\times \hat{\mathbf{H}}\left(z_{k}\right)^{-1} \boldsymbol{\Omega}\left(\mathbf{I}_{r}+\boldsymbol{\Omega}\right)^{-1} \mathbf{U}_{s}^{H} \mathbf{Q}\left(z_{k}\right)\right] \\
& \times\left(\mathbf{I}_{m}+\mathbf{P}\right)^{-1 / 2}
\end{aligned}
$$

with

$$
\begin{aligned}
\mathbf{Q}\left(z_{k}\right) & =\left(\frac{1}{K} \mathbf{Y} \mathbf{Y}^{H}-z_{k} \mathbf{I}_{m}\right)^{-1} \\
\hat{\mathbf{H}}\left(z_{k}\right) & =\mathbf{I}_{r}+z_{k} \boldsymbol{\Omega}\left(\mathbf{I}_{r}+\boldsymbol{\Omega}\right)^{-1} \mathbf{U}_{s}^{H} \mathbf{Q}\left(z_{k}\right) \mathbf{U}_{s}
\end{aligned}
$$

Then, replacing ( $\left.\hat{\mathbf{R}}-z_{k} \mathbf{I}_{m}\right)^{-1}$ by Eq.(48) in Eq.(44) and developing the obtained result, one obtains:

$$
\hat{\eta}\left(j_{1}, j_{2}\right)=D_{1}-D_{2}-D_{3}+D_{4}
$$

$$
\begin{aligned}
& \hat{\eta}\left(j_{1}, j_{2}\right)= \frac{1}{(2 i \pi)^{2}} \oint_{\mathcal{C}_{j_{1}}^{-}} \oint_{\mathcal{C}_{j_{2}}^{-}} \boldsymbol{s}_{1}^{H} \mathbf{E}\left(z_{1}\right) \mathbf{B E}\left(z_{2}\right) \boldsymbol{s}_{2} d z_{1} d z_{2} \\
&- \frac{1}{(2 i \pi)^{2}} \oint_{\mathcal{C}_{j_{1}}^{-}} \oint_{\mathcal{C}_{j_{2}}^{-}}\left[\hat{\mathbf{e}}_{1}^{H}\left(z_{1}\right) \hat{\mathbf{H}}\left(z_{1}\right)^{-1} \mathbf{\Omega}\left(\mathbf{I}_{r}+\mathbf{\Omega}\right)^{-1}\right. \\
&\left.\times \mathbf{U}_{s}^{H} \mathbf{Q}\left(z_{1}\right)\left(\mathbf{I}_{m}+\mathbf{P}\right)^{-1 / 2} \mathbf{B}\right] \mathbf{E}\left(z_{2}\right) \boldsymbol{s}_{2} d z_{1} d z_{2} \\
&- \frac{1}{(2 i \pi)^{2}} \oint_{\mathcal{C}_{j_{1}}^{-}} \oint_{\mathcal{C}_{j_{2}}^{-}} \boldsymbol{s}_{1}^{H} \mathbf{E}\left(z_{1}\right)\left[\mathbf{B}\left(\mathbf{I}_{m}+\mathbf{P}\right)^{-1 / 2} z_{2}\right. \\
&\left.\times \mathbf{Q}\left(z_{2}\right) \mathbf{U}_{s} \hat{\mathbf{H}}\left(z_{2}\right)^{-1} \hat{\mathbf{e}}_{2}\left(z_{2}\right)\right] d z_{1} d z_{2} \\
&+\frac{1}{(2 i \pi)^{2}} \oint_{\mathcal{C}_{j_{1}}^{-}} \oint_{\mathcal{C}_{j_{2}}^{-}} \hat{\mathbf{e}}_{1}^{H}\left(z_{1}\right) \hat{\mathbf{H}}\left(z_{1}\right)^{-1} \hat{\mathbf{C}}\left(z_{1}, z_{2}\right) \\
& \times \hat{\mathbf{H}}\left(z_{2}\right)^{-1} \hat{\mathbf{e}}_{2}\left(z_{2}\right) d z_{1} d z_{2}
\end{aligned}
$$


with

$$
\begin{aligned}
\mathbf{E}(z)= & \left(\mathbf{I}_{m}+\mathbf{P}\right)^{-1 / 2} \mathbf{Q}(z)\left(\mathbf{I}_{m}+\mathbf{P}\right)^{-1 / 2} \\
\hat{\mathbf{e}}_{1}^{H}(z)= & \boldsymbol{s}_{1}^{H}\left(\mathbf{I}_{m}+\mathbf{P}\right)^{-1 / 2} z \mathbf{Q}(z) \mathbf{U}_{s} \\
\hat{\mathbf{C}}\left(z_{1}, z_{2}\right)= & \boldsymbol{\Omega}\left(\mathbf{I}_{r}+\boldsymbol{\Omega}\right)^{-1} \mathbf{U}_{s}^{H} \mathbf{Q}\left(z_{1}\right)\left(\mathbf{I}_{m}+\mathbf{P}\right)^{-1 / 2} \mathbf{B} \\
& \times\left(\mathbf{I}_{m}+\mathbf{P}\right)^{-1 / 2} z_{2} \mathbf{Q}\left(z_{2}\right) \mathbf{U}_{s} \\
\hat{\mathbf{e}}_{2}(z)= & \boldsymbol{\Omega}\left(\mathbf{I}_{r}+\boldsymbol{\Omega}\right)^{-1} \mathbf{U}_{s}^{H} \mathbf{Q}(z)\left(\mathbf{I}_{m}+\mathbf{P}\right)^{-1 / 2} \boldsymbol{s}_{2}
\end{aligned}
$$

\section{Determination of the deterministic complex integral equiv-} alent

The convergence of the terms $D_{1}$ to $D_{4}$ has now to be studied. Some of them will tend to 0 and the remainder of the terms will tend to a deterministic integral equivalent.

Proposition 3: Let B be a $m \times m$ deterministic complex matrix with a uniformly bounded spectral norm for all $m$. Then, under (As1-As3, As4.S) and the spiked model, $\forall j_{1}, j_{2} \in$ $\llbracket 1, r \rrbracket, \hat{\eta}\left(j_{1}, j_{2}\right)-\eta\left(j_{1}, j_{2}\right) \stackrel{\text { a.s. }}{\longrightarrow} 0$ with

$$
\begin{array}{r}
\eta\left(j_{1}, j_{2}\right)=\frac{1}{(2 i \pi)^{2}} \oint_{\gamma_{j_{1}}^{-}} \oint_{\gamma_{j_{2}}^{-}} \mathbf{e}_{1}^{H}\left(z_{1}\right) \mathbf{H}\left(z_{1}\right)^{-1} \mathbf{C}\left(z_{1}, z_{2}\right) \\
\times \mathbf{H}\left(z_{2}\right)^{-1} \mathbf{e}_{2}\left(z_{2}\right) d z_{1} d z_{2}
\end{array}
$$

where $\gamma_{j}^{-}$is a deterministic negatively oriented circle only enclosing $\tau_{j}$ (cf. Eq.(25)) and

$$
\begin{aligned}
\mathbf{H}(z)= & \mathbf{I}_{r}+z \bar{b}_{m}(z) \boldsymbol{\Omega}\left(\mathbf{I}_{r}+\boldsymbol{\Omega}\right)^{-1} \\
\mathbf{e}_{1}^{H}(z)= & z \bar{b}_{m}(z) \boldsymbol{s}_{1}^{H}\left(\mathbf{I}_{m}+\mathbf{P}\right)^{-1 / 2} \mathbf{U}_{s} \\
\mathbf{C}\left(z_{1}, z_{2}\right)= & z_{2} \bar{b}_{m}\left(z_{1}\right) \bar{b}_{m}\left(z_{2}\right) \boldsymbol{\Omega}\left(\mathbf{I}_{r}+\boldsymbol{\Omega}\right)^{-1} \mathbf{U}_{s}^{H}\left(\mathbf{I}_{m}+\mathbf{P}\right)^{-1 / 2} \\
& \times \mathbf{B}\left(\mathbf{I}_{m}+\mathbf{P}\right)^{-1 / 2} \mathbf{U}_{s} \\
\mathbf{e}_{2}(z)= & \bar{b}_{m}(z) \boldsymbol{\Omega}\left(\mathbf{I}_{r}+\boldsymbol{\Omega}\right)^{-1} \mathbf{U}_{s}^{H}\left(\mathbf{I}_{m}+\mathbf{P}\right)^{-1 / 2} \boldsymbol{s}_{2}
\end{aligned}
$$

Proof: The function $\mathbf{E}(z)$ in $D_{1}, D_{2}$ and $D_{3}$ can be rewritten as:

$$
\mathbf{E}(z)=\left(\mathbf{I}_{m}+\mathbf{P}\right)^{-1 / 2} \sum_{n=1}^{m} \frac{\hat{\boldsymbol{v}}_{n} \hat{\boldsymbol{v}}_{n}^{H}}{\hat{\zeta}_{n}-z}\left(\mathbf{I}_{m}+\mathbf{P}\right)^{-1 / 2}
$$

where $\hat{\zeta}_{n}$ and $\hat{\boldsymbol{v}}_{n}$ are the eigenvalues and the eigenvectors of $\mathbf{Y} \mathbf{Y}^{H} / K$ respectively. Thus, $\mathbf{E}\left(z_{1}\right)$ (resp. $\mathbf{E}\left(z_{2}\right)$ ) has a single simple pole $\hat{\zeta}_{n}$. As a consequence, $\forall j_{1}, j_{2} \in \llbracket 1, r \rrbracket$, $\mathcal{C}_{j_{1}}^{-}$(resp. $\left.\mathcal{C}_{j_{2}}^{-}\right)$does not encompass $\mathbf{E}\left(z_{1}\right)$ (resp. $\mathbf{E}\left(z_{2}\right)$ ) under (As3, As4.S). Thus, $D_{1}=D_{2}=D_{3}=0$ and:

$$
\begin{array}{r}
\hat{\eta}\left(j_{1}, j_{2}\right)=\frac{1}{(2 i \pi)^{2}} \oint_{\mathcal{C}_{j_{1}}^{-}} \oint_{\mathcal{C}_{j_{2}}^{-}} \hat{\mathbf{e}}_{1}^{H}\left(z_{1}\right) \hat{\mathbf{H}}\left(z_{1}\right)^{-1} \hat{\mathbf{C}}\left(z_{1}, z_{2}\right) \\
\times \hat{\mathbf{H}}\left(z_{2}\right)^{-1} \hat{\mathbf{e}}_{2}\left(z_{2}\right) d z_{1} d z_{2}
\end{array}
$$

We will then determine a deterministic equivalent of Eq.(63), i.e. its convergence in the large dimensional regime. From lemma 5 of [32] and [22],

$$
\begin{aligned}
& \hat{\mathbf{H}}(z) \underset{m, K \rightarrow \infty}{\stackrel{\text { a.s. }}{\longrightarrow}} \mathbf{H}(z)=\mathbf{I}_{r}+z \bar{b}_{m}(z) \boldsymbol{\Omega}\left(\mathbf{I}_{r}+\boldsymbol{\Omega}\right)^{-1} \\
& m / K \rightarrow c<\infty \\
& \hat{\mathbf{e}}_{1}^{H}(z) \underset{m, K \rightarrow \infty}{\stackrel{\text { a.s. }}{\rightarrow}} \mathbf{e}_{1}^{H}(z)=z \bar{b}_{m}(z) \boldsymbol{s}_{1}^{H}\left(\mathbf{I}_{m}+\mathbf{P}\right)^{-1 / 2} \mathbf{U}_{s} \\
& m / K \rightarrow c<\infty \\
& \hat{\mathbf{e}}_{2}(z) \underset{m, K \rightarrow \infty}{\stackrel{\text { a.s. }}{\rightarrow}} \mathbf{e}_{2}(z)=\bar{b}_{m}(z) \boldsymbol{\Omega}\left(\mathbf{I}_{r}+\boldsymbol{\Omega}\right)^{-1} \mathbf{U}_{s}^{H}\left(\mathbf{I}_{m}+\mathbf{P}\right)^{-1 / 2} \boldsymbol{s}_{2} \\
& m / K \rightarrow c<\infty
\end{aligned}
$$

Then, the most difficult part concerns the convergence of

$$
\begin{aligned}
\hat{\mathbf{C}}\left(z_{1}, z_{2}\right)= & z_{2} \boldsymbol{\Omega}\left(\mathbf{I}_{r}+\boldsymbol{\Omega}\right)^{-1} \mathbf{U}_{s}^{H} \mathbf{Q}\left(z_{1}\right)\left(\mathbf{I}_{m}+\mathbf{P}\right)^{-1 / 2} \mathbf{B} \\
& \times\left(\mathbf{I}_{m}+\mathbf{P}\right)^{-1 / 2} \mathbf{Q}\left(z_{2}\right) \mathbf{U}_{s} \\
= & z_{2} \boldsymbol{\Omega}\left(\mathbf{I}_{r}+\boldsymbol{\Omega}\right)^{-1} \mathbf{U}_{s}^{H} \mathbf{Q}\left(z_{1}\right) \mathbf{D Q}\left(z_{2}\right) \mathbf{U}_{s}
\end{aligned}
$$

which contains the matrix $m \times m$ complex $\mathbf{Q}\left(z_{1}\right) \mathbf{D Q}\left(z_{2}\right)$. As the convergence cannot be addressed through classical methods as in [32], we focus on the asymptotic equivalence of matrices. Indeed, it can be proven using Gaussian methods [45], [46] that

$$
\mathbf{Q}\left(z_{1}\right) \mathbf{D Q}\left(z_{2}\right) \longleftrightarrow \bar{b}_{m}\left(z_{1}\right) \bar{b}_{m}\left(z_{2}\right) \mathbf{D}
$$

where $\longleftrightarrow$ means the asymptotic equivalence of two matrices in the large dimensional regime. However, the proof is a result in itself and therefore is out of scope of this paper. For this reason, it is omitted here. Consequently, considering Eq.(69),

$$
\begin{aligned}
\hat{\mathbf{C}}\left(z_{1}, z_{2}\right) \underset{\substack{m, K \rightarrow \infty \\
m / K \rightarrow c<\infty}}{\stackrel{\text { a.s. }}{\longrightarrow}} \mathbf{C}\left(z_{1}, z_{2}\right)= & z_{2} \bar{b}_{m}\left(z_{1}\right) \bar{b}_{m}\left(z_{2}\right) \boldsymbol{\Omega}\left(\mathbf{I}_{r}+\boldsymbol{\Omega}\right)^{-1} \mathbf{U}_{s}^{H} \\
& \times\left(\mathbf{I}_{m}+\mathbf{P}\right)^{-1 / 2} \mathbf{B}\left(\mathbf{I}_{m}+\mathbf{P}\right)^{-1 / 2} \mathbf{U}_{s}(70)
\end{aligned}
$$

As a result, $\hat{\eta}\left(j_{1}, j_{2}\right)-\eta\left(j_{1}, j_{2}\right) \stackrel{\text { a.s. }}{\longrightarrow} 0$ with

$$
\begin{array}{r}
\eta\left(j_{1}, j_{2}\right)=\frac{1}{(2 i \pi)^{2}} \oint_{\gamma_{j_{1}}^{-}} \oint_{\gamma_{j_{2}}^{-}} \mathbf{e}_{1}^{H}\left(z_{1}\right) \mathbf{H}\left(z_{1}\right)^{-1} \mathbf{C}\left(z_{1}, z_{2}\right) \\
\times \mathbf{H}\left(z_{2}\right)^{-1} \mathbf{e}_{2}\left(z_{2}\right) d z_{1} d z_{2}
\end{array}
$$

where $\gamma_{j}^{-}$is a deterministic negatively oriented circle only enclosing $\tau_{j}$ (cf. Eq.(25)).

\section{E. Determination of the expression of the deterministic equiv-} alent

Let us now find the expression of the deterministic equivalent $\eta\left(j_{1}, j_{2}\right)$ as a function of the eigenvalues and eigenvectors of the covariance matrix $\mathbf{R}$

Proposition 4: Let B be a $m \times m$ deterministic complex matrix with a uniformly bounded spectral norm for all $m$. Then, under (As1-As3, As4.S) and the spiked model,

$$
\eta\left(j_{1}, j_{2}\right)=\chi_{j_{1}} \chi_{j_{2}} \boldsymbol{s}_{1}^{H} \boldsymbol{\Pi}_{j_{1}} \mathbf{B} \boldsymbol{\Pi}_{j_{2}} \boldsymbol{s}_{2}
$$

with $\chi_{j}=\frac{1-c \omega_{j}^{-2}}{1+c \omega_{j}^{-1}}$ and $\left\{j_{1}, j_{2}\right\} \in \llbracket 1, r \rrbracket^{2}$.

Proof: We first decompose Eq.(71) as:

$$
\eta\left(j_{1}, j_{2}\right)=\frac{1}{2 i \pi} \oint_{\gamma_{j_{1}}^{-}} \mathbf{g}\left(z_{1}\right) d z_{1} \mathbf{B} \frac{1}{2 i \pi} \oint_{\gamma_{j_{1}}^{-}} \tilde{\mathbf{g}}\left(z_{2}\right) d z_{2}
$$

with

$$
\begin{aligned}
& \mathbf{g}(z)=\bar{b}_{m}(z) \mathbf{e}_{1}^{H}(z) \mathbf{H}(z)^{-1} \mathbf{\Omega}\left(\mathbf{I}_{r}+\mathbf{\Omega}\right)^{-1} \mathbf{U}_{s}^{H}\left(\mathbf{I}_{m}+\mathbf{P}\right)^{-1 / 2} \\
& \tilde{\mathbf{g}}(z)=z \bar{b}_{m}(z)\left(\mathbf{I}_{m}+\mathbf{P}\right)^{-1 / 2} \mathbf{U}_{s} \mathbf{H}(z)^{-1} \mathbf{e}_{2}(z)
\end{aligned}
$$

to distinguish the contributions associated to $z_{1}$ and $z_{2}$.

After an eigendecomposition of Eqs.(74) and (75) and, using [22], one obtains:

$$
\begin{aligned}
\mathbf{g}\left(z_{1}\right) & =\boldsymbol{s}_{1}^{H} \sum_{l=1}^{r} \frac{\omega_{l} \boldsymbol{\Pi}_{l}}{\left(1+\omega_{l}\right)^{2}} \frac{z_{1} \bar{b}_{m}^{2}\left(z_{1}\right)}{1+z_{1} \bar{b}_{m}\left(z_{1}\right) \frac{\omega_{l}}{1+\omega_{l}}} \\
\tilde{\mathbf{g}}\left(z_{2}\right) & =\sum_{l=1}^{r} \frac{\omega_{l} \boldsymbol{\Pi}_{l}}{\left(1+\omega_{l}\right)^{2}} \frac{z_{2} \bar{b}_{m}^{2}\left(z_{2}\right)}{1+z_{2} \bar{b}_{m}\left(z_{2}\right) \frac{\omega_{l}}{1+\omega_{l}}} \boldsymbol{s}_{2}
\end{aligned}
$$

As a result, from the properties of $\bar{b}_{m}(z)$ and residue calculus (see [22] for further details), one obtains:

$$
\eta\left(j_{1}, j_{2}\right)=\xi\left(\tau_{j_{1}}\right) \xi\left(\tau_{j_{2}}\right) \boldsymbol{s}_{1}^{H} \boldsymbol{\Pi}_{j_{1}} \mathbf{B} \boldsymbol{\Pi}_{j_{2}} \boldsymbol{s}_{2}
$$

with

$$
\xi\left(\tau_{j}\right)=\frac{\left(1+h\left(\tau_{j}\right)\right) \bar{b}_{m}\left(\tau_{j}\right)}{h^{\prime}\left(\tau_{j}\right)}
$$


Finally, the last step consists in expressing $\xi\left(\tau_{j}\right)$ as a function of $\omega_{j}$. Using Corollary 2 from [22], one expresses $\xi\left(\tau_{j}\right)$ as:

$$
\xi\left(\tau_{j}\right)=\chi_{j}=\frac{1-c \omega_{j}^{-2}}{1+c \omega_{j}^{-1}}
$$

As a consequence,

$$
\hat{\eta}\left(j_{1}, j_{2}\right) \underset{\substack{m, K \rightarrow \infty \\ m / K \rightarrow c<\infty}}{\stackrel{\text { a.s. }}{\longrightarrow}} \eta\left(j_{1}, j_{2}\right)=\chi_{j_{1}} \chi_{j_{2}} \boldsymbol{s}_{1}^{H} \boldsymbol{\Pi}_{j_{1}} \mathbf{B} \boldsymbol{\Pi}_{j_{2}} \boldsymbol{s}_{2}
$$

with $\left\{j_{1}, j_{2}\right\} \in \llbracket 1, r \rrbracket^{2}$.

\section{F. Convergence of the structured $Q F$}

From the development of the structured $\mathrm{QF}$, we recall that the convergences of the simple QFs $\boldsymbol{s}_{1}^{H} \hat{\boldsymbol{\Pi}}_{i} \mathbf{B} \boldsymbol{s}_{2}$ and $\boldsymbol{s}_{1}^{H} \mathbf{B} \hat{\boldsymbol{\Pi}}_{i} \boldsymbol{s}_{2}$, $\forall i \in \llbracket 1, r \rrbracket$ can be easily determined from [22]:

$$
\begin{gathered}
\boldsymbol{s}_{1}^{H} \hat{\boldsymbol{\Pi}}_{i} \mathbf{B} \boldsymbol{s}_{2} \underset{\substack{m, K \rightarrow \infty \\
m / K \rightarrow c<\infty}}{\stackrel{\text { a.s. }}{\longrightarrow}} \chi_{i} \boldsymbol{s}_{1}^{H} \boldsymbol{\Pi}_{i} \mathbf{B} \boldsymbol{s}_{2} \\
\boldsymbol{s}_{1}^{H} \mathbf{B} \hat{\boldsymbol{\Pi}}_{i} \boldsymbol{s}_{2} \underset{\substack{m, K \rightarrow \infty \\
m / K \rightarrow c<\infty}}{\stackrel{\text { a.s. }}{m}} \chi_{i} \boldsymbol{s}_{1}^{H} \mathbf{B} \boldsymbol{\Pi}_{i} \boldsymbol{s}_{2}
\end{gathered}
$$

where $\chi_{i}$ is defined as in Section III.C.

Then, also using Eq.(81) in Eq.(43), one easily obtains:

$$
\begin{aligned}
& \boldsymbol{s}_{1}^{H} \hat{\boldsymbol{\Pi}}_{\mathrm{c}}^{\perp} \mathbf{B} \hat{\boldsymbol{\Pi}}_{\mathrm{c}}^{\perp} \boldsymbol{s}_{2} \underset{\substack{m, K \rightarrow \infty \\
m / K \rightarrow c<\infty}}{\stackrel{\text { a.s. }}{\rightarrow}} \\
& \boldsymbol{s}_{1}^{H}\left[\mathbf{I}_{m}-\sum_{i=1}^{r} \chi_{i} \boldsymbol{\Pi}_{i}\right] \mathbf{B}\left[\mathbf{I}_{m}-\sum_{i=1}^{r} \chi_{i} \boldsymbol{\Pi}_{i}\right] \boldsymbol{s}_{2} \\
& \boldsymbol{s}_{1}^{H} \hat{\boldsymbol{\Pi}}_{\mathrm{c}}^{\perp} \mathbf{B} \hat{\boldsymbol{\Pi}}_{\mathrm{c}}^{\perp} \boldsymbol{s}_{2} \underset{\substack{m, K \rightarrow \infty \\
m / K \rightarrow c<\infty}}{\stackrel{\mathrm{a} . \mathrm{s}}{\longrightarrow}} \boldsymbol{s}_{1}^{H} \overline{\boldsymbol{\Pi}}_{\mathrm{c}, \mathrm{S}}^{\perp} \mathbf{B} \overline{\boldsymbol{\Pi}}_{\mathrm{c}, \mathrm{S}}^{\perp} \boldsymbol{s}_{2}
\end{aligned}
$$

with $\overline{\boldsymbol{\Pi}}_{\mathrm{c}, \mathrm{S}}^{\perp}=\sum_{i=1}^{m} \psi_{i} \boldsymbol{u}_{i} \boldsymbol{u}_{i}^{H}$ and

$$
\psi_{i}= \begin{cases}1, & \text { if } i>r \\ 1-\chi_{i}, & \text { if } i \leqslant r\end{cases}
$$

\section{REFERENCES}

[1] I. Reed, J. Mallett, and L. Brennan, "Rapid convergence rate in adaptive arrays," IEEE Trans. on Aero. and Elec. Syst., vol. AES-10, no. 6, pp. 853 - 863, November 1974.

[2] J. Ward, "Space-time adaptive processing for airborne radar," Lincoln Lab., MIT, Lexington, Mass., USA, Tech. Rep., December 1994.

[3] L. Scharf and B. Friedlander, "Matched subspace detectors," IEEE Trans. on Sig. Proc., vol. 42, pp. 2146 - 2157, 1994.

[4] R. Schmidt, "Multiple emitter location and signal parameter estimation," IEEE Trans.-ASSP, vol. 34, no. 3, pp. 276 - 280, March 1986.

[5] R. Roy and T. Kailath, "ESPRIT-estimation of signal parameters via rotational invariant techniques," IEEE Trans.-ASSP, vol. 37, no. 7, pp. 984 - 995, July 1989.

[6] I. Kirstein and D. Tufts, "Adaptive detection using a low rank approximation to a data matrix," IEEE Trans. on Aero. and Elec. Syst., vol. 30, pp. $55-67,1994$.

[7] A. Haimovich, "The eigencanceler: Adaptive radar by eigenanalysis methods," IEEE Trans. on Aero. and Elec. Syst., vol. 32, no. 2, pp. 532 - 542, April 1996.

[8] G. Ginolhac and G. Jourdain, “"principal component inverse" algorithm for detection in the presence of reverberation," IEEE Journal of Oceanic Engineering, vol. 27, no. 2, pp. 310 - 321, April 2002.

[9] M. Rangaswamy, F. Lin, and K. Gerlach, "Robust adaptive signal processing methods for heterogeneous radar clutter scenarios," Signal Processing, vol. 84, pp. 1653 - 1665, 2004.

[10] E. Kelly, "An adaptive detection algorithm," IEEE Trans. on Aero. and Elec. Syst., vol. 22, no. 1, pp. 115-127, March 1986.
[11] F. Robey, D. Fuhrmann, E. Kelly, and R. Nitzberg, "A CFAR adaptive matched filter detector," IEEE Trans. on Aero. and Elec. Syst., vol. 28, no. 2, pp. $208-216,1992$.

[12] S. Kraut, L. Scharf, and L. McWhorter, "Adaptive subspace detectors," IEEE Trans. on Sig. Proc., vol. 49, no. 1, pp. 1-16, january 2001.

[13] O. Besson and L. Scharf, "CFAR matched direction detector," IEEE Trans. on Sig. Proc., vol. 54, no. 7, pp. 2840 - 2845, July 2006.

[14] A. Haimovich, "Asymptotic distribution of the conditional signal-tonoise ratio in an eigenanalysis-based adaptive array," IEEE Trans. on Aero. and Elec. Syst., vol. 33, pp. 988 - 997, 1997.

[15] C. Peckham, A. Haimovich, T. Ayoub, J. Goldstein, and I. Reed, "Reduced-rank STAP performance analysis," IEEE Trans. on Aero. and Elec. Syst., vol. 36, no. 2, pp. 664 - 676, April 2000.

[16] G. Ginolhac, P. Forster, F. Pascal, and J.-P. Ovarlez, "Performance of two low-rank STAP filters in a heterogeneous noise," IEEE Trans. on Signal Process., vol. 61, pp. 57 - 61, 2013.

[17] H. Krim, P. Forster, and J. Proakis, "Operator approach to performance analysis of root-MUSIC and root-min-norm," IEEE Trans. on Sig. Proc., vol. 40, no. 7, pp. 1687 - 1696, July 1992.

[18] G. Ginolhac, P. Forster, F. Pascal, and J. Ovarlez, "Exploiting persymmetry for low-rank space time adaptive processing," Signal Processing, vol. 97, no. 4, pp. 242 - 251, April 2014.

[19] X. Mestre, "Improved estimation of eigenvalues and eigenvectors of covariance matrices using their sample estimates," IEEE Transactions on Information Theory, vol. 54, no. 11, pp. 5113 - 5129, November 2008.

[20] — - "On the asymptotics behavior of the sample estimates of eigenvalues and eigenvectors of covariance matrices," IEEE Transactions on Signal Processing, vol. 56, no. 11, pp. 5353 - 5368, November 2009.

[21] P. Vallet, P. Loubaton, and X. Mestre, "Improved subspace estimation for multivariate observations of high dimension the deterministic signals case," IEEE Trans. on Information Theory, vol. 58, no. 2, pp. 1043 1068, Feb. 2012.

[22] R. Couillet and W. Hachem, "Fluctuations of spiked random matrix models and failure diagnosis in sensor networks," IEEE Transactions on Information Theory, vol. 59, no. 1, pp. 509 - 525, 2013.

[23] X. Mestre and M. Lagunas, "Modified subspace algorithms for doa estimation with large arrays," IEEE Transactions on Information Theory, vol. 56, no. 2, pp. 598 - 614, February 2008.

[24] R. Couillet, F. Pascal, and J. Silverstein, "Robust estimates of covariance matrices in the large dimensional regime," IEEE Transactions on Information Theory, vol. 60, no. 11, pp. 7269 - 7278, 2014, http://arxiv.org/abs/1204.5320.

[25] R. Nadakuditi and J. Silverstein, "Fundamental limit of sample generalized eigenvalue based detection of signals in noise using relatively few signal-bearing and noise-only samples," IEEE Journal of Selected Topics in Signal Processing, vol. 4, no. 3, pp. 468 - 480, 2010.

[26] N. Asendorf and R. Nadakuditi, "The performance of a matched subspace detector that uses subspaces estimated from finite, noisy, training data," IEEE Trans. on Sig. Proc., vol. 61, no. 8, pp. 1972 - 1985, April 2013.

[27] A. Combernoux, F. Pascal, G. Ginolhac, and M. Lesturgie, "Performances of low rank detectors based on random matrix theory with application to stap," RADAR, Oct. 2014.

[28] - "Asymptotic performance of the low rank adaptive normalized matched filter in a large dimensional regime," ICASSP, Apr. 2015, accepted.

[29] I. Johnstone, "On the distribution of the largest principal component," The Annals of Statistics, vol. 29, no. 2, pp. 295 - 327, 2001.

[30] F. Benaych-Georges and R. Nadakuditi, "The eigenvalues and eigenvectors of finite, low rank perturbations of large random matrices," $A d v$. in Math., vol. 227, no. 1, pp. $494-521,2011$.

[31] D. Paul, "Asymptotics of sample eigenstructure for a large dimensional spiked covariance model," Statistica Sinica, vol. 17, no. 4, pp. 1617 $1642,2007$.

[32] W. Hachem, P. Loubaton, X. Mestre, J. Najim, and P. Vallet, "A subspace estimator of fixed rank perturbations of large random matrices," Journal of Multivariate Analysis, vol. 114, pp. 427 - 447, 2013.

[33] B. Tang, J. Tang, and Y. Peng, "Performance of knowledge aided space time adaptive processing," IET Radar Sonar Navig., vol. 5, no. 3, pp. $331-340,2010$.

[34] — - "Clutter nulling performance of SMI in amplitude heterogeneous clutter environments," IEEE Trans. on Aero. and Elec. Syst., vol. 49, no. 2, pp. 1366 - 1373, April 2013.

[35] X. Mestre and M. Lagunas, "Finite sample size effect on minimum variance beamformers: Optimum diagonal loading factor for large arrays," 
IEEE Transactions on Signal Processing, vol. 54, no. 1, pp. 69 - 82, January 2006.

[36] F. Rubio, X. Mestre, and W. Hachem, "A CLT on the SNR of diagonally loaded MVDR filters," IEEE Trans. on Sig. Proc., vol. 60, no. 8, pp. 4178 - 4195, August 2012.

[37] J. Yu, F. Rubio, and M. McKay, "Performance analysis of minimum variance asset allocation with high frequency data," ICASSP, pp. 6496 - 6500, May 2013.

[38] V. Marčenko and L. Pastur, "Distributions of eigenvalues for somme set of random matrices," Math USSR-Sbornik, vol. 1, no. 4, pp. 457 - 483, April 1967.

[39] J. Baik and J. W. Silverstein, "Eigenvalues of large sample covariance matrices of spiked population models," Journal of Multivariate Analysis, vol. 97, pp. 1643 - 1697, 2006.

[40] P. Billingsley, Probability and Measure, 3rd ed. New York, NY: Wiley, 1995.

[41] V. Girko, An Introduction to Statistical Analysis of Random Arrays. VSP International Science Publishers, 1998, ch. 14 Ten years of general statistical analysis, http://www.general-statisticalanalysis.girko.freewebspace.com/chapter14.pdf.

[42] J. Li and P. Stoica, MIMO Radar Signal Processing, 1st ed. Wiley, 2009.

[43] D. Tse and P. Viswanath, Fundamentals of Wireless Communication, 1st ed. Cambridge University Press, 2005.

[44] L. Hormander, An Introduction to Complex Analysis in Several Variables, ser. North-Holland Mathematical Library. Amsterdam: NorthHolland, 1973.

[45] R. Couillet and M. Debbah, Random Matrix Methods for Wireless Communications. Cambridge University Press, November 2011, http://hal.archives-ouvertes.fr/hal-00658725.

[46] W. Hachem, O. Khorunzhy, P. Loubaton, J. Najim, and L. Pastur, "A new approach for capacity analysis of large dimensional multi-antenna channels," IEEE Trans. on Inf. Th., 2008. 